Research Article

\title{
A Semilinear Wave Equation with a Boundary Condition of Many-Point Type: Global Existence and Stability of Weak Solutions
}

\author{
Giai Giang Vo \\ Department of Mathematics, Faculty of Information Technology, Huflit University, 155 Su Van Hanh Street, District 10, \\ Ho Chi Minh City 700000, Vietnam \\ Correspondence should be addressed to Giai Giang Vo; g.giangvo@gmail.com \\ Received 19 July 2015; Accepted 28 September 2015 \\ Academic Editor: Khalil Ezzinbi \\ Copyright (C) 2015 Giai Giang Vo. This is an open access article distributed under the Creative Commons Attribution License, which \\ permits unrestricted use, distribution, and reproduction in any medium, provided the original work is properly cited. \\ This paper is devoted to the study of a wave equation with a boundary condition of many-point type. The existence of weak solutions \\ is proved by using the Galerkin method. Also, the uniqueness and the stability of solutions are established.
}

\section{Introduction}

Recently, initial-boundary value problems of wave equations have appeared more and more in mechanics, they have been deeply studied by many authors, and we can refer to the works [1-13]. In this paper, we consider the following nonlinear wave equation with a boundary condition of many-point type:

$$
\begin{aligned}
& u_{t t}-\rho u_{x x}+f(x, u)=0, \quad 0<x<1,0<t<T, \\
& u(1, t)=0 \text {, } \\
& \rho u_{x}(0, t) \\
& =\int_{0}^{t} k(t-s) h\left(u\left(\xi_{0}, s\right), u\left(\xi_{1}, s\right), \ldots, u\left(\xi_{N}, s\right)\right) d s \\
& +g\left(u\left(\xi_{0}, t\right)\right)+\sigma(t), \\
& u(x, 0)=u_{0}(x), \\
& u_{t}(x, 0)=u_{1}(x),
\end{aligned}
$$

where $f, g, h, k, \sigma, u_{0}, u_{1}$ are given real functions and $\rho, \xi_{1}$, $\xi_{2}, \ldots, \xi_{N}$ are positive constants such that $0=\xi_{0}<\xi_{1}<\cdots<$ $\xi_{N}<1$.
Dang and Alain [4] studied the global existence of the following problem:

$$
\begin{aligned}
u_{t t}-u_{x x}+\left|u_{t}\right|^{\alpha} \operatorname{sign}\left(u_{t}\right) & =0, \\
u(1, t) & =0, \\
u_{x}(0, t) & =g(t), \\
u(x, 0) & =u_{0}(x), \\
u_{t}(x, 0) & =u_{1}(x),
\end{aligned}
$$

where $0<\alpha<1$ is a constant and $g, u_{0}, u_{1}$ are given functions.

In [11], Santos considered the following problem:

$$
\begin{aligned}
u_{t t}-\rho(t) u_{x x} & =0, \\
u(0, t) & =0, \\
u(1, t)+\int_{0}^{t} g(t-s) \rho(s) u_{x}(1, s) d s & =0, \\
u(x, 0) & =u_{0}(x), \\
u_{t}(x, 0) & =u_{1}(x),
\end{aligned}
$$

in which $g, \rho, u_{0}, u_{1}$ are given functions. He studied the asymptotic behavior of the solutions of problem (3) with 
respect to the time variable. In this case, problem (3) is a mathematical model for a linear one-dimensional motion of an elastic bar connected with a viscoelastic element at an end of the bar.

Applying the Mikusinski operational calculus, D. Takači and A. Takači [12] gave the formula for finding the exact solutions of the linear wave equation given by

$$
\begin{aligned}
u_{t t}-u_{x x}+K u+\lambda u_{t} & =f(x, t), \\
u_{x}(0, t) & =v(t), \\
-u_{x}(1, t) & =\delta u(1, t)+\varepsilon u_{t}(1, t), \\
u(x, 0) & =u_{0}(x), \\
u_{t}(x, 0) & =u_{1}(x),
\end{aligned}
$$

where $K, \lambda, \delta, \varepsilon, h, \omega$ are given nonnegative constants and $f, g, u_{0}, u_{1}$ are given functions. Also, the unknown function $u(x, t)$ and the unknown boundary value $v(t)$ satisfy the following integral equation:

$$
\begin{aligned}
v(t)= & g(t)+h u(0, t) \\
& -\omega h \int_{0}^{t} \sin (\omega(t-s)) u(0, s) d s .
\end{aligned}
$$

It is worth noting that the function $v(t)$ is deduced from a Cauchy problem for an ordinary differential equation at the boundary condition $t=0$. Indeed, if $v(t)$ satisfies the following Cauchy problem

$$
\begin{aligned}
& v^{\prime \prime}(t)+2 p v^{\prime}(t)+p_{0} v(t) \\
& =q u_{t t}(0, t)+q_{0} u_{t}(0, t)+q_{1} u(0, t), \\
& v(0)=v_{0}, \\
& v^{\prime}(0)=v_{1},
\end{aligned}
$$

where $p, p_{0}, q, q_{0}, q_{1}, v_{0}, v_{1}$ are constants such that $p_{0}>p^{2}$, we can easily show that

$$
v(t)=g(t)+h u(0, t)+\int_{0}^{t} k(t-s) u(0, s) d s,
$$

where

$$
\begin{aligned}
& g(t)=\exp (-p t)\left[v_{1}-p v_{0}+p q u_{0}(0)-q u_{1}(0)\right] \frac{\sin \omega t}{\omega} \\
& +\exp (-p t)\left\{\left[v_{0}-q u_{0}(0)\right] \cos \omega t-\frac{q_{0}}{\omega} u_{0}(0)\right\}, \\
& k(t)=\exp (-p t)\left\{\left[q\left(p^{2}-\omega^{2}\right)-p q_{0}+q_{1}\right] \frac{\sin \omega t}{\omega}\right. \\
& \left.\quad+\left(q_{0}-2 p q\right) \cos \omega t\right\},
\end{aligned}
$$

with $\omega=\sqrt{p_{0}-p^{2}}, h=q+q_{0} / \omega$. In the special case of $p=$ $q_{0}=q_{1}=0$, we obtain (5).
Besides, Nguyen and Giang Vo [8] obtained the asymptotic behavior of the weak solution of the following initialboundary value problem as $\varepsilon \rightarrow 0_{+}$:

$$
\begin{aligned}
u_{t t}-u_{x x}+K u+\lambda u_{t} & =f(x, t), \\
u(1, t) & =0, \\
u_{x}(0, t) & =g(t)+h u(0, t)+\varepsilon u_{t}(0, t) \\
& \quad-\int_{0}^{t} k(t-s) u(0, s) d s, \\
u(x, 0) & =u_{0}(x), \\
u_{t}(x, 0) & =u_{1}(x),
\end{aligned}
$$

in which $K, \lambda, h \in \mathbb{R}, \varepsilon>0$ are given constants and $f, g, k, u_{0}, u_{1}$ are given functions. Problem (9) is said to be a mathematical model describing a shock problem involving a linear viscoelastic bar.

The organization of this paper is as follows. First of all, we establish the global existence and uniqueness of weak solutions of problem (1). The proof is based on the Galerkin method associated with a priori estimates and the weak compact method. Finally, here we prove that this solution is stable in the sense of continuous dependence on the given data $(f, g, h, k, \sigma)$. This paper is a relative generalization of the works $[4,6,11,12]$.

\section{Preliminaries}

For convenience, we denote by $\langle\cdot, \cdot\rangle$ and $\|\cdot\|$, respectively, the scalar product and the norm in $L^{2}(0,1)$. Also, we define a closed subspace of the Sobolev space $H^{1}(0,1)$ as follows:

$$
H=\left\{u \in H^{1}(0,1): u(1)=0\right\},
$$

with the following scalar product and norm:

$$
\begin{gathered}
\langle u, v\rangle_{H}=\left\langle u_{x}, v_{x}\right\rangle, \\
\|u\|_{H}=\left\|u_{x}\right\| .
\end{gathered}
$$

Then it is easy to show the following.

Lemma 1. The embedding $H \hookrightarrow C^{0}([0,1])$ is compact and

$$
\begin{aligned}
& \|u\|_{C^{0}([0,1])} \leq\|u\|_{H} \leq\|u\|_{H^{1}(0,1)} \leq \sqrt{2}\|u\|_{H} \text {, } \\
& \forall u \in H
\end{aligned}
$$

On the other hand, we also have the following result.

Lemma 2. Let $\varepsilon>0$. Then

$$
\|v\|_{C^{0}([0,1])}^{2} \leq \varepsilon\left\|v_{x}\right\|^{2}+\left(1+\frac{1}{\varepsilon}\right)\|v\|^{2},
$$

$$
\forall u \in H^{1}(0,1) .
$$

The proof of the lemma is straightforward; we omit the details. 
Remark 3. Let $\varepsilon=1$, we get

$$
\begin{aligned}
\|v\|_{C^{0}([0,1])} \leq\left(\left\|v_{x}\right\|^{2}+2\|v\|^{2}\right)^{1 / 2} \leq \sqrt{2}\|v\|_{H^{1}(0,1)}, & \\
\forall u & \in H^{1}(0,1) .
\end{aligned}
$$

Next, if $W$ is a real Banach space with norm $\|\cdot\|_{W}$, $L^{p}(0, T ; W)$ consists of equivalence classes of strongly measurable functions $u:(0, T) \rightarrow W$ such that $\|u\|_{L^{p}(0, T ; W)}<\infty$, with

$$
\begin{aligned}
& \|u\|_{L^{p}(0, T ; W)} \\
& = \begin{cases}\left(\int_{0}^{T}\|u(t)\|_{W}^{p} d t\right)^{1 / p}, & \text { if } 1 \leq p<\infty, \\
\underset{0<t<T}{\operatorname{ess} \sup _{0}\|u(t)\|_{W},} & \text { if } p=\infty .\end{cases}
\end{aligned}
$$

It is not difficult to prove that $L^{p}(0, T ; W)$ is a Banach space.

Let $W_{0}, W_{1}$, and $W_{2}$ be Banach spaces satisfying $W_{0} \subset$ $W_{1} \subset W_{2}$. We further assume that $W_{0}$ and $W_{2}$ are reflexive, and the imbedding $W_{0} \hookrightarrow W_{1}$ is compact. Set

$$
\begin{aligned}
W & (0, T) \\
& =\left\{u: u \in L^{p}\left(0, T ; W_{0}\right), u_{t} \in L^{q}\left(0, T ; W_{2}\right)\right\},
\end{aligned}
$$

where $1 \leq p, q \leq \infty$. Then $W(0, T)$ is a Banach space with the norm

$$
\|u\|_{W(0, T)}=\|u\|_{L^{p}\left(0, T ; W_{0}\right)}+\left\|u_{t}\right\|_{L^{q}\left(0, T ; W_{2}\right)} .
$$

We also have the following lemma.

Lemma 4 (see [14]). Let $1<p, q<\infty$. The embedding $W(0, T) \hookrightarrow L^{p}\left(0, T ; W_{1}\right)$ is compact.

\section{Global Existence and Uniqueness of Weak Solutions}

To investigate the existence of a unique weak solution of problem (1), the following assumptions are needed:

$$
\begin{aligned}
& \left(A_{1}\right) u_{0} \in H \text { and } u_{1} \in L^{2}(0,1) \\
& \left(A_{2}\right) \sigma \in H^{1}(0, T), k \in W^{1,1}(0, T) \\
& \left(A_{3}\right) f, D_{2} f \in C^{0}([0,1] \times \mathbb{R}) \text { satisfy the following condi- } \\
& \text { tions: }
\end{aligned}
$$

(i) There exist positive functions $\sigma_{1}, \sigma_{2} \in L^{1}(0,1)$ such that

$$
\int_{0}^{u} f(x, s) d s \geq-\sigma_{1}(x) u^{2}-\sigma_{2}(x),
$$

$$
\text { a.e. } x \in[0,1], \forall u \in \mathbb{R} \text {. }
$$

(ii) There exist positive functions $\widehat{\sigma} \in L^{2}(0,1)$ and $\widehat{f} \in C^{0}(\mathbb{R})$ such that

$$
\left|D_{2} f(x, u)\right| \leq \widehat{\sigma}(x) \widehat{f}(u), \quad \text { a.e. } x \in[0,1], \forall u \in \mathbb{R} .
$$

$\left(A_{4}\right) g \in C^{2}(\mathbb{R})$; there exist constants $a, b>0$ such that

$$
\int_{0}^{u} g(s) d s \geq-a u^{2}-b, \quad \forall u \in \mathbb{R} .
$$

$\left(A_{5}\right) h \in C^{0}\left(\mathbb{R}^{N+1}\right)$; there exist constants $c, d>0$ such that

$$
\left|h\left(u_{0}, u_{1}, \ldots, u_{N}\right)\right| \leq c \sum_{i=0}^{N}\left|u_{i}\right|+d,
$$

$$
\forall u_{i} \in \mathbb{R}, i=\overline{0, N} \text {. }
$$

$\left(A_{6}\right)$ For each $M>0$, there exists a constant $c_{M}>0$ such that

$$
\begin{array}{r}
\left|h\left(u_{0}, \ldots, u_{N}\right)-h\left(v_{0}, \ldots, v_{N}\right)\right| \leq c_{M} \sum_{i=0}^{N}\left|u_{i}-v_{i}\right| \\
\forall\left|u_{i}\right|,\left|v_{i}\right| \leq M, i=\overline{0, N} .
\end{array}
$$

With these assumptions, we have the following theorem.

Theorem 5. Let assumptions $\left(A_{1}\right)-\left(A_{6}\right)$ hold. Then problem (1) has a unique weak solution $u$ such that

$$
\begin{gathered}
u \in L^{\infty}(0, T ; H), \\
u_{t} \in L^{\infty}\left(0, T ; L^{2}(0,1)\right), \\
u\left(\xi_{i}, \cdot\right) \in H^{1}(0, T), \quad i=\overline{0, N} .
\end{gathered}
$$

Remark 6. In the special case of $N=0$ and $g^{\prime}(t)>-1$, for all $t \in \mathbb{R}$, we have obtained the same results in the paper [6].

Proof of Theorem 5. The main tool of this proof is the Galerkin method. The procedure includes four steps as follows:

(i) Galerkin approximation.

(ii) A priori estimates.

(iii) Limiting process.

(iv) Uniqueness of the weak solutions.

Step 1 (Galerkin approximation). We use a special orthonormal base of $H$ :

$$
\begin{aligned}
\varphi_{k}(x) & =\sqrt{\frac{2}{\left(1+\mu_{k}^{2}\right)}} \cos \left(\mu_{k} x\right), \\
\mu_{k} & =(2 k-1) \frac{\pi}{2},
\end{aligned}
$$

Now we are looking for the approximate solution of problem (1) in the form

$$
u^{m}(x, t)=\sum_{k=1}^{m} \omega_{m k}(t) \varphi_{k}(x),
$$


where the coefficient functions $\omega_{m k}(t)$ satisfy the following system of nonlinear differential equations

$$
\begin{gathered}
\left\langle u_{t t}^{m}(\cdot, t), \varphi_{k}\right\rangle+\rho\left\langle u^{m}(\cdot, t), \varphi_{k}\right\rangle_{H}+v_{m}(t) \varphi_{k}(0) \\
+\left\langle f\left(\cdot, u^{m}(\cdot, t)\right), \varphi_{k}\right\rangle=0, \quad k=\overline{1, m},
\end{gathered}
$$

with

$$
\begin{aligned}
& v_{m}(t)=\int_{0}^{t} k(t-s) \\
& \cdot h\left(u^{m}\left(\xi_{0}, s\right), u^{m}\left(\xi_{1}, s\right), \ldots, u^{m}\left(\xi_{N}, s\right)\right) d s \\
& \quad+g\left(u^{m}\left(\xi_{0}, t\right)\right)+\sigma(t), \\
& u^{m}(\cdot, 0) \equiv u_{0}^{m}=\sum_{k=1}^{m} a_{m k} \varphi_{k} \longrightarrow u_{0}
\end{aligned}
$$$$
\text { strongly in } H^{1}(0,1) \text {, }
$$$$
u_{t}^{m}(\cdot, 0) \equiv u_{1}^{m}=\sum_{k=1}^{m} b_{m k} \varphi_{k} \longrightarrow u_{1}
$$

strongly in $L^{2}(0,1)$.

By substituting $\rho_{k}=\sqrt{\rho} \mu_{k}$, we can rewrite the system of (26)(29) as follows:

$$
\begin{aligned}
& \omega_{m k}^{\prime \prime}(t)+\rho_{k}^{2} \omega_{m k}(t)=-\frac{1}{\left\|\varphi_{k}\right\|^{2}}\left[v_{m}(t) \varphi_{k}(0)\right. \\
& \left.\quad+\left\langle f\left(\cdot, u^{m}(\cdot, t)\right), \varphi_{k}\right\rangle\right], \\
& v_{m}(t)=\int_{0}^{t} k(t-s) \\
& \quad \cdot h\left(u^{m}\left(\xi_{0}, s\right), u^{m}\left(\xi_{1}, s\right), \ldots, u^{m}\left(\xi_{N}, s\right)\right) d s \\
& \quad+g\left(u^{m}\left(\xi_{0}, t\right)\right)+\sigma(t), \\
& \omega_{m k}(0)=a_{m k}, \\
& \omega_{m k}^{\prime}(0)=b_{m k},
\end{aligned}
$$

$$
k=\overline{1, m} .
$$

Therefore, we obtain

$$
\begin{gathered}
\omega_{m k}(t)=a_{m k} \cos \left(\rho_{k} t\right)+b_{m k} \frac{\sin \left(\rho_{k} t\right)}{\rho_{k}}-\frac{2}{\varphi_{k}(0)} \int_{0}^{t} d s \\
\cdot \int_{0}^{s} \frac{\sin \left[\rho_{k}(t-s)\right]}{\rho_{k}} k(s-\tau) \\
\cdot h\left(u^{m}\left(\xi_{0}, \tau\right), \ldots, u^{m}\left(\xi_{N}, \tau\right)\right) d \tau-\frac{2}{\varphi_{k}(0)}
\end{gathered}
$$

$$
\begin{array}{r}
\cdot \int_{0}^{t} \frac{\sin \left[\rho_{k}(t-s)\right]}{\rho_{k}}\left[\sigma(s)+g\left(u^{m}\left(\xi_{0}, s\right)\right)\right] d s \\
-\frac{2}{\varphi_{k}^{2}(0)} \int_{0}^{t} \frac{\sin \left[\rho_{k}(t-s)\right]}{\rho_{k}}\left\langle f\left(\cdot, u^{m}(s)\right), \varphi_{k}\right\rangle d s, \\
k=\overline{1, m} .
\end{array}
$$

Applying the Schauder fixed-point theorem, it is not difficult that system $(31)$ has a solution $\left(\omega_{m 1}, \omega_{m 2}, \ldots, \omega_{m m}\right)$ on an interval $\left[0, T_{m}\right]$. This implies that in system (26)-(29) there exists a solution to $u^{m}(t)$ on $\left[0, T_{m}\right]$. Moreover, we can extend the approximate solution $u^{m}$ to the whole interval $[0, T]$ (see [15]).

Step 2 (a priori estimates). In (26), we replace $\varphi_{k}(x)$ by $u_{t}^{m}(x, t)$. Then integrating from 0 to $t$, we have after some calculations

$$
\begin{aligned}
& E_{m}(t)=E_{m}(0)+2 \sigma(0) u_{0}^{m}(0)+2 \int_{0}^{u_{0}^{m}(0)} g(s) d s \\
& +2 \int_{0}^{1} d x \int_{0}^{u_{0}^{m}(x)} f(x, s) d s-2 \sigma(t) u^{m}(0, t) \\
& +2 \int_{0}^{t} \sigma^{\prime}(s) u^{m}(0, s) d s-2 \int_{0}^{u^{m}(0, t)} g(s) d s \\
& -2 \int_{0}^{1} \int_{0}^{u^{m}(x, t)} f(x, s) d s d x-2 \int_{0}^{t} u_{t}^{m}(0, r) d r \\
& . \int_{0}^{r} k(r-s) h\left(u^{m}\left(\xi_{0}, s\right), \ldots, u^{m}\left(\xi_{N}, s\right)\right) d s \\
& \equiv E_{m}(0)+2 \sigma(0) u_{0}^{m}(0)+2 \int_{0}^{u_{0}^{m}(0)} g(s) d s \\
& +2 \int_{0}^{1} d x \int_{0}^{u_{0}^{m}(x)} f(x, s) d s+\sum_{k=1}^{5} I_{k}(t),
\end{aligned}
$$

where

$$
E_{m}(t)=\left\|u_{t}^{m}(\cdot, t)\right\|^{2}+\rho\left\|u^{m}(\cdot, t)\right\|_{H}^{2} \cdot
$$

We will estimate, respectively, the following terms on the right-hand side of (32).

Estimating $I_{1}(t)$. Using (33) and Lemma 1, we infer that

$$
\begin{aligned}
\left|u^{m}(0, t)\right| & \leq\left\|u^{m}(\cdot, t)\right\|_{C^{0}([0,1])} \leq\left\|u^{m}(\cdot, t)\right\|_{H} \\
& \leq \frac{1}{\sqrt{\rho}} \sqrt{E_{m}(t)} .
\end{aligned}
$$

Hence,

$$
\begin{aligned}
I_{1}(t) & =-2 \sigma(t) u^{m}(0, t) \leq \varepsilon\left|u^{m}(0, t)\right|^{2}+\frac{1}{\varepsilon} \sigma^{2}(t) \\
& \leq \varepsilon E_{m}(t)+\frac{1}{\varepsilon}\|\sigma\|_{L^{\infty}(0, T)}^{2}, \quad \forall \varepsilon>0 .
\end{aligned}
$$


Estimating $I_{2}(t)$. Using Lemma 1 and inequality (34), we arrive at

$$
\begin{aligned}
I_{2}(t) & =2 \int_{0}^{t} \sigma^{\prime}(s) u^{m}(0, s) d s \\
& \leq \frac{1}{\rho} \int_{0}^{t}\left|\sigma^{\prime}(s)\right| d s+\rho \int_{0}^{t}\left|\sigma^{\prime}(s)\right|\left|u^{m}(0, s)\right|^{2} d s \\
& \leq \int_{0}^{t}\left|\sigma^{\prime}(s)\right| E_{m}(s) d s+\frac{1}{\rho}\left\|\sigma^{\prime}\right\|_{L^{1}(0, T)} .
\end{aligned}
$$

Estimating $I_{3}(t)$. From assumption $\left(A_{4}\right)$, we have

$$
I_{3}(t)=-2 \int_{0}^{u^{m}(0, t)} g(s) d s \leq 2 a\left|u^{m}(0, t)\right|^{2}+2 b .
$$

On the other hand, we see that

$$
\begin{aligned}
\left\|u^{m}(\cdot, t)\right\|^{2} & =\left\|u_{0}^{m}+\int_{0}^{t} u_{t}^{m}(\cdot, s) d s\right\|^{2} \\
& \leq 2\left\|u_{0}^{m}\right\|^{2}+2 t \int_{0}^{t} E_{m}(s) d s .
\end{aligned}
$$

It follows from (37), (38), and Lemma 2 that

$$
\begin{aligned}
I_{3}(t) \leq & 2 \varepsilon \frac{a}{\rho} E_{m}(t)+4 a t\left(1+\frac{1}{\varepsilon}\right) \int_{0}^{t} E_{m}(s) d s \\
& +4 a\left(1+\frac{1}{\varepsilon}\right)\left\|u_{0}^{m}\right\|^{2}+2 b .
\end{aligned}
$$

Estimating $I_{4}(t)$. Owing to assumption $\left(A_{3}\right)$-(i), (38), and Lemma 2, it is not difficult to show that

$$
\begin{aligned}
I_{4}(t)= & -2 \int_{0}^{1} d x \int_{0}^{u^{m}(x, t)} f(x, s) d s \\
\leq & 2 \int_{0}^{1} \sigma_{1}(x)\left|u^{m}(x, t)\right|^{2} d x+2\left\|\sigma_{2}\right\|_{L^{1}(0,1)} \\
\leq & \varepsilon \frac{2}{\rho}\left\|\sigma_{1}\right\|_{L^{1}(0,1)} E_{m}(t) \\
& +4 t\left(1+\frac{1}{\varepsilon}\right)\left\|\sigma_{1}\right\|_{L^{1}(0,1)} \int_{0}^{t} E_{m}(s) d s \\
& +4\left(1+\frac{1}{\varepsilon}\right)\left\|u_{0}^{m}\right\|^{2}\left\|\sigma_{1}\right\|_{L^{1}(0,1)}+2\left\|\sigma_{2}\right\|_{L^{1}(0,1)} .
\end{aligned}
$$

Estimating $I_{5}(t)$. Applying integration by parts, it follows that

$$
\begin{aligned}
& I_{5}(t)=-2 \int_{0}^{t} u_{t}^{m}(0, r) d r \\
& \cdot \int_{0}^{r} k(r-s) h\left(u^{m}\left(\xi_{0}, s\right), \ldots, u^{m}\left(\xi_{N}, s\right)\right) d s \\
& =-2 u^{m}(0, t) \\
& \cdot \int_{0}^{t} k(t-s) h\left(u^{m}\left(\xi_{0}, s\right), \ldots, u^{m}\left(\xi_{N}, s\right)\right) d s \\
& +2 k(0) \\
& \quad \cdot \int_{0}^{t} u^{m}(0, s) h\left(u^{m}\left(\xi_{0}, s\right), \ldots, u^{m}\left(\xi_{N}, s\right)\right) d s \\
& +2 \int_{0}^{t} u^{m}(0, r) d r \\
& \quad \cdot \int_{0}^{r} k^{\prime}(r-s) h\left(u^{m}\left(\xi_{0}, s\right), \ldots, u^{m}\left(\xi_{N}, s\right)\right) d s \\
& \equiv J_{1}(t)+J_{2}(t)+J_{3}(t) .
\end{aligned}
$$

We can estimate the integrals in the right-hand side of (41) as follows:

$$
\begin{aligned}
& J_{1}(t)=-2 u^{m}(0, t) \\
& \cdot \int_{0}^{t} k(t-s) h\left(u^{m}\left(\xi_{0}, s\right), \ldots, u^{m}\left(\xi_{N}, s\right)\right) d s \\
& \leq 2 c\left|u^{m}(0, t)\right| \sum_{i=0}^{N} \int_{0}^{t}|k(t-s)|\left|u^{m}\left(\xi_{i}, s\right)\right| d s \\
& +2 d\left|u^{m}(0, t)\right| \int_{0}^{t}|k(t-s)| d s \leq 2(N+1) \frac{c}{\sqrt{\rho}} \\
& \cdot \sqrt{E_{m}(t)} \int_{0}^{t}|k(t-s)| \sqrt{E_{m}(s)} d s+2 \frac{d}{\sqrt{\rho}} \\
& \text { - } \sqrt{E_{m}(t)} \int_{0}^{t}|k(s)| d s \leq 2 \varepsilon E_{m}(t)+(N+1)^{2} \\
& \cdot \frac{c^{2}}{\varepsilon \rho}\|k\|_{L^{2}(0, T)}^{2} \int_{0}^{t} E_{m}(s) d s+\frac{d^{2}}{\varepsilon \rho}\|k\|_{L^{1}(0, T)}^{2}, \\
& J_{2}(t)=2 k(0) \\
& \text {. } \int_{0}^{t} u^{m}(0, s) h\left(u^{m}\left(\xi_{0}, s\right), \ldots, u^{m}\left(\xi_{N}, s\right)\right) d s \\
& \leq 2(N+1) \frac{c}{\rho}|k(0)| \int_{0}^{t} E_{m}(s) d s+2 \frac{d}{\sqrt{\rho}}|k(0)| \\
& \cdot \int_{0}^{t} \sqrt{E_{m}(s)} d s \leq\left[1+2(N+1) \frac{c}{\rho}|k(0)|\right] \\
& \cdot \int_{0}^{t} E_{m}(s) d s+\frac{d^{2}}{\rho} T k^{2}(0),
\end{aligned}
$$




$$
\begin{aligned}
& J_{3}(t)=2 \int_{0}^{t} u^{m}(0, r) d r \\
& \cdot \int_{0}^{r} k^{\prime}(r-s) h\left(u^{m}\left(\xi_{0}, s\right), \ldots, u^{m}\left(\xi_{N}, s\right)\right) d s \\
& \leq 2(N+1) \frac{c}{\rho} \int_{0}^{t} \sqrt{E_{m}(r) d r} \\
& \cdot \int_{0}^{r}\left|k^{\prime}(r-s)\right| \sqrt{E_{m}(s)} d s+2 \frac{d}{\sqrt{\rho}} \int_{0}^{t} \sqrt{E_{m}(r) d r} \\
& \cdot \int_{0}^{r}\left|k^{\prime}(r-s)\right| d s \leq 2(N+1) \frac{c}{\rho}\left[\int_{0}^{t} E_{m}(r) d r\right. \\
& \left.+\int_{0}^{t}\left(\int_{0}^{r}\left|k^{\prime}(r-s)\right| \sqrt{E_{m}(s)} d s\right)^{2} d r\right] \\
& +\int_{0}^{t} E_{m}(r) d r+\frac{d^{2} t}{\rho}\left\|k^{\prime}\right\|_{L^{1}(0, T)}^{2} \cdot
\end{aligned}
$$

Going in for the Cauchy-Schwartz inequality, we arrive at

$$
\begin{aligned}
& \left(\int_{0}^{r}\left|k^{\prime}(r-s)\right| \sqrt{E_{m}(s)} d s\right)^{2} \\
& \quad \leq \int_{0}^{r}\left|k^{\prime}(r-s)\right| d s \int_{0}^{r}\left|k^{\prime}(r-s)\right| E_{m}(s) d s \\
& \quad \leq\left\|k^{\prime}\right\|_{L^{1}(0, T)} \int_{0}^{r}\left|k^{\prime}(r-s)\right| E_{m}(s) d s .
\end{aligned}
$$

Consequently,

$$
\begin{aligned}
& \int_{0}^{t}\left(\int_{0}^{r}\left|k^{\prime}(r-s)\right| \sqrt{E_{m}(s)} d s\right)^{2} d r \\
& \quad \leq\left\|k^{\prime}\right\|_{L^{1}(0, T)} \int_{0}^{t} d r \int_{0}^{r}\left|k^{\prime}(r-s)\right| E_{m}(s) d s \\
& \quad=\left\|k^{\prime}\right\|_{L^{1}(0, T)} \int_{0}^{t} E_{m}(s) d s \int_{s}^{t}\left|k^{\prime}(r-s)\right| d r \\
& \quad \leq\left\|k^{\prime}\right\|_{L^{1}(0, T)}^{2} \int_{0}^{t} E_{m}(s) d s .
\end{aligned}
$$

It follows from (44) and (46) that

$$
\begin{aligned}
& J_{3}(t) \\
& \qquad \leq\left[1+2(N+1) \frac{c}{\rho}\left(1+\left\|k^{\prime}\right\|_{L^{1}(0, T)}^{2}\right)\right] \int_{0}^{t} E_{m}(r) d r \\
& \quad+\frac{d^{2} T}{\rho}\left\|k^{\prime}\right\|_{L^{1}(0, T)}^{2} .
\end{aligned}
$$

We deduce from the estimates of $J_{1}(t), J_{2}(t), J_{3}(t)$ that

$$
I_{5}(t) \leq 2 \varepsilon E_{m}(t)+D_{T}^{1} \int_{0}^{t} E_{m}(s) d s+D_{T}^{2}
$$

with

$$
\begin{aligned}
D_{T}^{1}= & (N+1)^{2} \frac{c^{2}}{\varepsilon \rho}\|k\|_{L^{2}(0, T)}^{2} \\
& +2(N+1) \frac{c}{\rho}\left(\left\|k^{\prime}\right\|_{L^{1}(0, T)}^{2}+|k(0)|+1\right)+2, \\
D_{T}^{2}= & \frac{d^{2}}{\varepsilon \rho}\left[\varepsilon T\left\|k^{\prime}\right\|_{L^{1}(0, T)}^{2}+\|k\|_{L^{1}(0, T)}^{2}+\varepsilon T k^{2}(0)\right] .
\end{aligned}
$$

On the other hand, by (28), (29), and assumptions $\left(A_{1}\right)-\left(A_{3}\right)$,

$$
\begin{gathered}
E_{m}(0)+2 \sigma(0) u_{0}^{m}(0)+2 \int_{0}^{u_{0}^{m}(0)} g(s) d s \\
+2 \int_{0}^{1} d x \int_{0}^{u_{0}^{m}(x)} f(x, s) d s \leq C,
\end{gathered}
$$

where $C$ is a positive constant depending only on $u_{0}, u_{1}, f, g, \sigma$.

Combining (32), (35), (36), (39), (40), (48), and (50), we obtain after some rearrangements

$$
E_{m}(t) \leq \varepsilon D_{T}^{3} E_{m}(t)+\int_{0}^{t} D_{T}^{4}(s) E_{m}(s) d s+D_{T}^{5},
$$

where

$$
\begin{aligned}
D_{T}^{3}= & \frac{2}{\rho}\left\|\sigma_{1}\right\|_{L^{1}(0,1)}+2 \frac{a}{\rho}+3 \\
D_{T}^{4}(s)= & \left|\sigma^{\prime}(s)\right|+4 T\left(1+\frac{1}{\varepsilon}\right)\left(\left\|\rho_{1}\right\|_{L^{1}(0,1)}+a\right)+D_{T}^{1}, \\
D_{T}^{5}= & \frac{1}{\varepsilon}\|\sigma\|_{L^{\infty}(0, T)}^{2}+\frac{1}{\rho}\left\|\sigma^{\prime}\right\|_{L^{1}(0, T)}+2\left\|\sigma_{2}\right\|_{L^{1}(0,1)} \\
& +4\left(1+\frac{1}{\varepsilon}\right)\left(\left\|\sigma_{1}\right\|_{L^{1}(0,1)}+a\right)\left\|u_{0}^{m}\right\|^{2} \\
& +D_{T}^{2}+C+2 b .
\end{aligned}
$$

Choosing $\varepsilon D_{T}^{3}=1 / 2$, by Gronwall's inequality, we have

$$
E_{m}(t) \leq 2 D_{T}^{5} \exp \left(2 \int_{0}^{t} D_{T}^{4}(s) d s\right) \leq D_{T}
$$

where $D_{T}$ is a positive constant depending on $T$.

Next, we will require the following lemma.

Lemma 7. There exists a positive constant $C_{T}$ depending only on $T$ such that

$$
\int_{0}^{t}\left|u_{t}^{m}\left(\xi_{i}, s\right)\right|^{2} d s \leq C_{T}, \quad \forall t \in[0, T], i=\overline{0, N}
$$


Proof of Lemma 7. We put

$$
\begin{aligned}
k_{m i}(t) & =\sum_{k=1}^{m} \cos \left(\mu_{k} \xi_{i}\right) \frac{\sin \left(\rho_{k} t\right)}{\rho_{k}}, \\
g_{m i}(t) & =\sum_{k=1}^{m} \varphi_{k}\left(\xi_{i}\right)\left[a_{m k} \cos \left(\rho_{k} t\right)+b_{m k} \frac{\sin \left(\rho_{k} t\right)}{\rho_{k}}\right] \\
-2 \sum_{k=1}^{m} \frac{\cos \left(\mu_{k} \xi_{i}\right)}{\varphi_{k}(0)} & \int_{0}^{t} \frac{\sin \left[\rho_{k}(t-s)\right]}{\rho_{k}}\left\langle f\left(\cdot, u^{m}(\cdot, s)\right), \varphi_{k}\right\rangle d s .
\end{aligned}
$$

In view of $(25)$ and $(31), u^{m}\left(\xi_{i}, t\right)$ can be rewritten as follows:

$$
u^{m}\left(\xi_{i}, t\right)=g_{m i}(t)-2 \int_{0}^{t} k_{m i}(t-s) v_{m}(s) d s .
$$

In connection with $g_{m i}(t)$, we have the following lemma.

Lemma 8. There exists a positive constant $\widehat{C}_{T}$ depending only on $T$ such that

$$
\int_{0}^{t}\left|g_{m i}^{\prime}(s)\right|^{2} d s \leq \widehat{C}_{T}, \quad \forall t \in[0, T], i=\overline{0, N} .
$$

Proof of Lemma 8. We define

$$
g_{m i}^{\prime}(t)=-p_{m i}(t)+q_{m i}(t)-r_{m i}(t)-s_{m i}(t),
$$

with

$$
\begin{aligned}
& p_{m i}(t)=\sum_{k=1}^{m} \varphi_{k}(0) \rho_{k} a_{m k} \cos \left(\mu_{k} \xi_{i}\right) \sin \left(\rho_{k} t\right), \\
& q_{m i}(t)=\sum_{k=1}^{m} \varphi_{k}(0) b_{m k} \cos \left(\mu_{k} \xi_{i}\right) \cos \left(\rho_{k} t\right), \\
& r_{m i}(t)=2 \sum_{k=1}^{m} \frac{\cos \left(\mu_{k} \xi_{i}\right)}{\varphi_{k}(0)} \frac{\sin \left(\rho_{k} t\right)}{\rho_{k}}\left\langle f\left(\cdot, u_{0}^{m}\right), \varphi_{k}\right\rangle, \\
& s_{m i}(t)=2 \sum_{k=1}^{m} \frac{\cos \left(\mu_{k} \xi_{i}\right)}{\varphi_{k}(0)} \\
& \cdot \int_{0}^{t} \frac{\sin \left[\rho_{k}(t-s)\right]}{\rho_{k}}\left\langle D_{2} f\left(\cdot, u^{m}(\cdot, s)\right)\right. \\
& \left.\cdot u_{t}^{m}(\cdot, s), \varphi_{k}\right\rangle d s .
\end{aligned}
$$

On the other hand, use the inequality

$$
(a+b+c+d)^{2} \leq 4\left(a^{2}+b^{2}+c^{2}+d^{2}\right),
$$

$\forall a, b, c, d \in \mathbb{R}$.

Hence,

$$
\begin{aligned}
\int_{0}^{t}\left|g_{m i}^{\prime}(s)\right|^{2} d s & \leq 4 \sum_{j \in\{p, q, r, s\}} \int_{0}^{t}\left|j_{m i}(s)\right|^{2} d s \\
& \equiv K_{1}(t)+K_{2}(t)+K_{3}(t)+K_{4}(t) .
\end{aligned}
$$

We will estimate each term on the right-hand side of this inequality.

Estimating $K_{1}(t)=4 \int_{0}^{t}\left|p_{m i}(s)\right|^{2} d s$. Thanks to (60) and (65),

$$
\begin{aligned}
& K_{1}(t)=\rho \int_{0}^{t}\left\{\sum_{k=1}^{m} \varphi_{k}(0)\right. \\
& \left.\cdot \mu_{k} a_{m k}\left\{\sin \left[\mu_{k}\left(\sqrt{\rho} s+\xi_{i}\right)\right]+\sin \left[\mu_{k}\left(\sqrt{\rho} s-\xi_{i}\right)\right]\right\}\right\}^{2} d s \\
& \leq 2 \rho \int_{0}^{t}\left\{\sum_{k=1}^{m} \varphi_{k}(0) \mu_{k} a_{m k} \sin \left[\mu_{k}\left(\sqrt{\rho} s+\xi_{i}\right)\right]\right\}^{2} d s \\
& +2 \rho \int_{0}^{t}\left\{\sum_{k=1}^{m} \varphi_{k}(0) \mu_{k} a_{m k} \sin \left[\mu_{k}\left(\sqrt{\rho} s-\xi_{i}\right)\right]\right\}^{2} d s \\
& \quad=2 \sqrt{\rho} \int_{\xi_{i}}^{\sqrt{\rho} t+\xi_{i}}\left[\sum_{k=1}^{m} \varphi_{k}(0) \mu_{k} a_{m k} \sin \left(\mu_{k} s\right)\right]^{2} d s \\
& +2 \sqrt{\rho} \int_{-\xi_{i}}^{\sqrt{\rho} t-\xi_{i}}\left[\sum_{k=1}^{m} \varphi_{k}(0) \mu_{k} a_{m k} \sin \left(\mu_{k} s\right)\right]^{2} d s .
\end{aligned}
$$

Now, we will need the following lemma.

Lemma 9. Let $a, b \in \mathbb{R}, a<b$ and $c_{k} \in \mathbb{R}, k=\overline{1, m}$. Then

$$
\begin{aligned}
& \int_{a}^{b}\left[\sum_{k=1}^{m} c_{k} \sin \left(\mu_{k} s\right)\right]^{2} d s \\
& \quad \leq 2(\max \{|a|,|b|\}+2) \int_{0}^{1}\left[\sum_{k=1}^{m} c_{k} \sin \left(\mu_{k} s\right)\right]^{2} d s
\end{aligned}
$$

The proof of this lemma is simple; we omit the details. Applying Lemma 9, we deduce from (28), (66), and assumption $\left(A_{1}\right)$ that

$$
\begin{aligned}
& K_{1}(t) \leq 8 \sqrt{\rho}\left(\sqrt{\rho} T+\xi_{i}+2\right) \\
& \cdot \int_{0}^{1}\left[\sum_{k=1}^{m} \varphi_{k}(0) \mu_{k} a_{m k} \sin \left(\mu_{k} s\right)\right]^{2} d s \\
& \leq 8 \sqrt{\rho}\left(\sqrt{\rho} T+\xi_{i}+2\right)\left\|u_{0 m}\right\|_{H}^{2} \leq C_{T},
\end{aligned}
$$

where $C_{T}$ always indicates a constant depending on $T$.

Estimating $K_{2}(t)=4 \int_{0}^{t}\left|q_{m i}(s)\right|^{2} d s$. Similarly, we also obtain

$$
K_{2}(t) \leq 8\left(T+\frac{\xi_{i}+2}{\sqrt{\rho}}\right)\left\|u_{1 m}\right\|^{2} \leq C_{T}
$$


Estimating $K_{3}(t)=4 \int_{0}^{t}\left|r_{m i}(s)\right|^{2} d s$. We see that

$$
\begin{aligned}
& r_{m i}(s)=\frac{2}{\sqrt{\rho}} \sum_{k=1}^{m} \frac{1}{\mu_{k}} \int_{0}^{1} \sin \left(\mu_{k} \sqrt{\rho} s\right) \cos \left(\mu_{k} \xi_{i}\right) \cos \left(\mu_{k} x\right) \\
& \cdot f\left(x, u_{0}^{m}(x)\right) d x=\frac{1}{2 \sqrt{\rho}} \\
& \cdot \int_{0}^{1} \sum_{k=1}^{m} \frac{1}{\mu_{k}}\left\{\sin \left[\mu_{k}\left(\sqrt{\rho} s+\xi_{i}+x\right)\right]\right. \\
& \left.+\sin \left[\mu_{k}\left(\sqrt{\rho} s-\xi_{i}-x\right)\right]\right\} f\left(x, u_{0}^{m}(x)\right) d x \\
& +\frac{1}{2 \sqrt{\rho}} \int_{0}^{1} \sum_{k=1}^{m} \frac{1}{\mu_{k}}\left\{\sin \left[\mu_{k}\left(\sqrt{\rho} s+\xi_{i}-x\right)\right]\right. \\
& \left.+\sin \left[\mu_{k}\left(\sqrt{\rho} s-\xi_{i}+x\right)\right]\right\} f\left(x, u_{0}^{m}(x)\right) d x .
\end{aligned}
$$

To estimate $K_{3}(t)$, we need the following lemma.

Lemma 10. Let $m \in \mathbb{N}$. One always has

$$
S_{m}(z)=\left|\sum_{k=0}^{m} \frac{\sin \mu_{k} z}{\mu_{k}}\right|<1+\frac{4}{\pi}, \quad \forall z \in \mathbb{R} .
$$

Proof of Lemma 10. First, we assume that $0<z \leq 1$. Set $M=$ $\left[z^{-1}\right]$ (which is an integer part of $z^{-1}$ ). We consider two cases of $m$.

Case $1(m>M+1)$. Then

$$
\begin{aligned}
S_{m}(z) & \leq \sum_{k=1}^{M} \frac{\left|\sin \mu_{j} z\right|}{\mu_{j}}+\left|\sum_{k=M+1}^{m} \frac{\sin \mu_{k} z}{\mu_{k}}\right| \\
& \equiv S_{m}^{1}(z)+S_{m}^{2}(z) .
\end{aligned}
$$

Since $|\sin \varphi| \leq|\varphi|$, for all $\varphi \in \mathbb{R}$, we get

$$
S_{m}^{1}(z)=\sum_{k=1}^{M} \frac{\left|\sin \mu_{k} z\right|}{\mu_{k}} \leq \sum_{k=1}^{M} \frac{\mu_{k} z}{\mu_{k}}=M z \leq 1 .
$$

Moreover, the function $\varphi \mapsto(\sin \varphi) / \varphi$ is decreasing on $(0, \pi / 2]$; hence, $\sin (\pi / 2) \varphi \geq \varphi$; for all $\varphi \in[0,1]$, it follows that

$$
\begin{aligned}
& \left|\sum_{i=k}^{m} \sin \mu_{i} z\right| \\
& \quad=\left|\frac{\sin ((m+k-1) / 2) \pi z \sin ((m-k+1) / 2) \pi z}{\sin (\pi z / 2)}\right| \\
& \quad \leq \frac{1}{\sin (\pi z / 2)} \leq \frac{1}{z}, \quad k=\overline{1, m}
\end{aligned}
$$

On the other hand, it is easy to verify the following equality by the induction:

$$
\begin{aligned}
\sum_{k=M+1}^{m} \frac{\sin \mu_{k} z}{\mu_{k}} & \\
= & \frac{1}{\mu_{M+1}} \sum_{k=M+1}^{m} \sin \mu_{k} z \\
& +\sum_{k=M+1}^{m-1}\left[\left(\frac{1}{\mu_{k+1}}-\frac{1}{\mu_{k}}\right) \sum_{i=k+1}^{m} \sin \mu_{i} z\right] .
\end{aligned}
$$

Using (74) and (75), we arrive at

$$
\begin{aligned}
S_{m}^{2}(z)= & \left|\sum_{k=M+1}^{m} \frac{\sin \mu_{k} z}{\mu_{k}}\right| \\
\leq & \frac{1}{\mu_{M+1}}\left|\sum_{k=M+1}^{m} \sin \mu_{k} z\right| \\
& +\sum_{k=M+1}^{m-1}\left[\left(\frac{1}{\mu_{k}}-\frac{1}{\mu_{k+1}}\right)\left|\sum_{i=k+1}^{m} \sin \mu_{i} z\right|\right] \\
\leq & \frac{1}{\mu_{M+1}} \frac{1}{z}+\sum_{k=M+1}^{m-1}\left(\frac{1}{\mu_{k}}-\frac{1}{\mu_{k+1}}\right) \frac{1}{z} \\
= & \left(\frac{2}{\mu_{M+1}}-\frac{1}{\mu_{m}}\right) \frac{1}{z}<\frac{4}{\pi} .
\end{aligned}
$$

Consequently, it follows from (72), (73), and (76) that

$$
S_{m}(z) \leq S_{m}^{1}(z)+S_{m}^{2}(z)<1+\frac{4}{\pi} .
$$

Case $2(1 \leq m \leq M+1)$. We have

$$
\begin{aligned}
S_{m}(z) & \leq\left|\sum_{k=1}^{m} \frac{\sin \mu_{k} z}{\mu_{k}}\right| \leq \sum_{k=1}^{M+1} \frac{\left|\sin \mu_{k} z\right|}{\mu_{k}} \leq \sum_{k=1}^{M+1} \frac{\mu_{k} z}{\mu_{k}} \\
& =(M+1) z<1+\frac{4}{\pi} .
\end{aligned}
$$

Combining (77) and (78), we conclude that

$$
S_{m}(z)<1+\frac{4}{\pi}, \quad \forall m \in \mathbb{N}, z \in(0,1] .
$$

Since $S_{m}(0)=0$ and the function $S_{m}$ is even, periodic with the period 2, thus inequality (79) holds for all $m \in \mathbb{N}, z \in \mathbb{R}$. The proof of Lemma 10 is complete.

By (70) and Lemma 10, it leads to

$$
\begin{aligned}
\left|r_{m i}(s)\right| & \leq \frac{1}{2 \sqrt{\rho}} \int_{0}^{1} 4\left(1+\frac{4}{\pi}\right)\left|f\left(x, u_{0}^{m}(x)\right)\right| d x \\
& =\frac{2}{\sqrt{\rho}}\left(1+\frac{4}{\pi}\right)\left\|f\left(\cdot, u_{0}^{m}\right)\right\|_{L^{1}(0,1)} .
\end{aligned}
$$


Hence, it follows from (28), (80), and assumptions $\left(A_{1}\right),\left(A_{3}\right)$ that

$$
\begin{aligned}
K_{3}(t) & =4 \int_{0}^{t}\left|r_{m i}(s)\right|^{2} d s \\
& \leq \frac{16 T}{\rho}\left(1+\frac{4}{\pi}\right)^{2}\left\|f\left(\cdot, u_{0}^{m}\right)\right\|_{L^{1}(0,1)}^{2} \leq C_{T} .
\end{aligned}
$$

Estimating $K_{4}(t)=4 \int_{0}^{t}\left|s_{m i}(s)\right|^{2} d s$. Proving in the same way as (81), we get

$$
\begin{gathered}
K_{4}(t)=4 \int_{0}^{t}\left|s_{m i}(s)\right|^{2} d s \leq \frac{8 T^{2}}{\rho}\left(1+\frac{4}{\pi}\right)^{2} \\
\cdot \int_{0}^{t}\left\|D_{2} f\left(\cdot, u^{m}(\cdot, s)\right) u_{t}^{m}(\cdot, s)\right\|_{L^{1}(0,1)}^{2} d s .
\end{gathered}
$$

On the other hand, using assumption $\left(A_{3}\right)$-(ii), then

$$
\left|D_{2} f\left(x, u^{m}(x, s)\right)\right| \leq \widehat{\sigma}(x) \sup _{|z| \leq \widehat{D}_{T}} \widehat{f}(z)=\widehat{C}_{T} \widehat{\sigma}(x),
$$

with $\widehat{D}_{T}=\sqrt{D_{T} / \rho}$.

Applying the Cauchy-Schwartz inequality, we clearly get

$$
\begin{aligned}
K_{4}(t) & \leq \frac{8 T^{2}}{\rho}\left(1+\frac{4}{\pi}\right)^{2} \widehat{C}_{T}^{2}\|\widehat{\sigma}\|^{2} \int_{0}^{t}\left\|u_{t}^{m}(\cdot, s)\right\|^{2} d s \\
& \leq C_{T} .
\end{aligned}
$$

Combining (65), (68), (69), (81), and (84), we obtain Lemma 8.

Remark 11. Lemma 3 in [4] is a special case of Lemma 8 with $g=h=0$ and $\rho=1$.

We now return to the proof of Lemma 7.

Note that it follows from (27) that

$$
\begin{aligned}
& v_{m}^{\prime}(t)=\sigma^{\prime}(t)+g^{\prime}\left(u^{m}\left(\xi_{0}, t\right)\right) u_{t}^{m}\left(\xi_{0}, t\right)+k(0) \\
& \cdot h\left(u^{m}\left(\xi_{0}, t\right), u^{m}\left(\xi_{1}, t\right), \ldots, u^{m}\left(\xi_{N}, t\right)\right) \\
& +\int_{0}^{t} k^{\prime}(t-s) \\
& \cdot h\left(u^{m}\left(\xi_{0}, s\right), u^{m}\left(\xi_{1}, t\right), \ldots, u^{m}\left(\xi_{N}, s\right)\right) d s .
\end{aligned}
$$

On account of (53) and assumptions $\left(A_{2}\right),\left(A_{4}\right)$, and $\left(A_{5}\right)$, we get

$$
\begin{aligned}
\left|v_{m}^{\prime}(t)\right| \leq & \left|\sigma^{\prime}(t)\right|+\sup _{|z| \leq \widehat{D}_{T}}\left|g^{\prime}(z)\right|\left|u_{t}^{m}\left(\xi_{0}, t\right)\right| \\
& +\left(|k(0)|+\left\|k^{\prime}\right\|_{L^{1}(0, T)}\right)\left[(N+1) c \widehat{D}_{T}+d\right] \\
\leq & \left|\sigma^{\prime}(t)\right|+C_{T}\left(\left|u_{t}^{m}\left(\xi_{0}, t\right)\right|+1\right) .
\end{aligned}
$$

Therefore, it is easy to see that

$$
\left|v_{m}^{\prime}(t)\right|^{2} \leq 2\left|\sigma^{\prime}(t)\right|^{2}+4 C_{T}^{2}\left(\left|u_{t}^{m}\left(\xi_{0}, t\right)\right|^{2}+1\right) .
$$

Applying Lemma 10 and the imbedding $H^{1}(0,1) \hookrightarrow$ $C^{0}([0,1])$, we deduce from (27) and (57) that

$$
\begin{aligned}
& \left|u_{t}^{m}\left(\xi_{i}, t\right)\right|=\mid g_{m i}^{\prime}(t)-2 k_{m i}(t) v_{m}(0) \\
& \quad-2 \int_{0}^{t} k_{m i}(t-s) v_{m}^{\prime}(s) d s|\leq| g_{m i}^{\prime}(t) \mid+\frac{2}{\sqrt{\rho}}(1 \\
& \left.\quad+\frac{4}{\pi}\right)\left|v_{m}(0)\right|+\frac{2}{\sqrt{\rho}}\left(1+\frac{4}{\pi}\right) \int_{0}^{t}\left|v_{m}^{\prime}(s)\right| d s \\
& \quad \leq\left|g_{m i}^{\prime}(t)\right|+C_{T}\left(1+\int_{0}^{t}\left|v_{m}^{\prime}(s)\right| d s\right) .
\end{aligned}
$$

Thus,

$$
\begin{aligned}
\left|u_{t}^{m}\left(\xi_{i}, t\right)\right|^{2} \leq & 2\left|g_{m i}^{\prime}(t)\right|^{2} \\
& +4 C_{T}^{2}\left(1+t \int_{0}^{t}\left|v_{m}^{\prime}(s)\right|^{2} d s\right) .
\end{aligned}
$$

Using the Cauchy-Schwartz inequality and Lemma 8, then we obtain from (87) and (89) that

$$
\begin{aligned}
& \int_{0}^{t}\left|u_{t}^{m}\left(\xi_{i}, s\right)\right|^{2} d s \\
& \leq 4 C_{T}^{2} t+2 \int_{0}^{t}\left|g_{m i}^{\prime}(s)\right|^{2} d s \\
& \quad+4 C_{T}^{2} \int_{0}^{t} \tau d \tau \int_{0}^{\tau}\left|v_{m}^{\prime}(s)\right|^{2} d s \\
& \leq 4 C_{T}^{2} t+\frac{16}{3} C_{T}^{4} t^{3}+4 C_{T}^{2} t\left\|\sigma^{\prime}\right\|_{L^{2}(0, T)}^{2} \\
& \quad+2 \int_{0}^{t}\left|g_{m i}^{\prime}(s)\right|^{2} d s \\
& \quad+16 C_{T}^{4} \int_{0}^{t} \tau d \tau \int_{0}^{\tau}\left|u_{t}^{m}\left(\xi_{0}, s\right)\right|^{2} d s \\
& \leq C_{T}+C_{T} \int_{0}^{t} d \tau \int_{0}^{\tau}\left|u_{t}^{m}\left(\xi_{0}, s\right)\right|^{2} d s .
\end{aligned}
$$

Hence,

$$
\begin{aligned}
& \sum_{i=0}^{N} \int_{0}^{t}\left|u_{t}^{m}\left(\xi_{i}, s\right)\right|^{2} d s \\
& \quad \leq(N+1) C_{T}\left[1+\int_{0}^{t}\left(\sum_{i=0}^{N} \int_{0}^{\tau}\left|u_{t}^{m}\left(\xi_{i}, s\right)\right|^{2} d s\right) d \tau\right] .
\end{aligned}
$$

By the Gronwall inequality, we get Lemma 7. This completes the proof of Lemma 7. 
Step 3 (limiting process). Due to (53) and (54), applying the Banach-Alaoglu theorem, we can extract a subsequence of sequence $\left\{u^{m}\right\}$, still labeled by the same notations, such that

$$
\begin{aligned}
u^{m} & \longrightarrow u \quad \text { weakly }{ }^{*} \text { in } L^{\infty}\left(0, T ; H^{1}(0,1)\right), \\
u_{t}^{m} & \longrightarrow u_{t} \quad \text { weakly }{ }^{*} \text { in } L^{\infty}\left(0, T ; L^{2}(0,1)\right), \\
u^{m}\left(\xi_{i}, \cdot\right) & \longrightarrow u\left(\xi_{i}, \cdot\right) \quad \text { weakly in } H^{1}(0, T) .
\end{aligned}
$$

Thanks to Lemma 4 and the compactness of the imbedding $H^{1}(0, T) \hookrightarrow C^{0}([0, T])$, they lead us to

$$
u^{m} \longrightarrow u
$$

strongly in $L^{2}((0,1) \times(0, T))$, a.e. in $(0,1) \times(0, T)$,

$u^{m}\left(\xi_{i}, \cdot\right) \longrightarrow u\left(\xi_{i}, \cdot\right) \quad$ strongly in $C^{0}([0, T])$.

By $(93)_{2}$ and assumptions $\left(A_{2}\right),\left(A_{4}\right)$, and $\left(A_{6}\right)$, we have

$$
\begin{aligned}
v_{m}(t) \longrightarrow & \int_{0}^{t} k(t-s) h\left(u\left(\xi_{0}, s\right), \ldots, u\left(\xi_{N}, s\right)\right) d s \\
& +g\left(u\left(\xi_{0}, t\right)\right)+\sigma(t)=v(t),
\end{aligned}
$$

strongly in $C^{0}([0, T])$.

Also, we apply the inequality

$$
\begin{aligned}
& \left|f\left(x, u^{m}(x, t)\right)-f(x, u(x, t))\right| \\
& \quad \leq d_{T}\left|u^{m}(x, t)-u(x, t)\right|
\end{aligned}
$$

where $d_{T}=\left\|D_{2} f\right\|_{C^{0}\left([0,1] \times\left[-\widehat{D}_{T}, \widehat{D}_{T}\right]\right)}$. By $(93)_{1}$ and (95), we get

$$
f\left(\cdot, u^{m}\right) \longrightarrow f(\cdot, u)
$$

$$
\text { strongly in } L^{2}((0,1) \times(0, T)) \text {. }
$$

Passing to the limit in (26) by $(92)_{1,2},(94)$, and (96), we have $u$ satisfying the equation

$$
\begin{gathered}
\frac{d}{d t}\left\langle u_{t}(\cdot, t), \varphi\right\rangle+\rho\langle u(\cdot, t), \varphi\rangle_{H}+v(t) \varphi(0) \\
+\langle f(\cdot, u(\cdot, t)), \varphi\rangle=0,
\end{gathered}
$$

for all $\varphi \in H$, a.e. $t \in[0, T]$.

On the other hand, it is easy to show a similar way as in $[4$, p. 588]

$$
\begin{gathered}
u(x, 0)=u_{0}(x), \\
u_{t}(x, 0)=u_{1}(x) .
\end{gathered}
$$

The existence of global solutions is proved.

Step 4 (uniqueness of the weak solutions). Let $u_{1}$ and $u_{2}$ be two weak solutions of problem (1). Then $u=u_{1}-u_{2}$ is a weak solution of the following problem:

$$
\begin{aligned}
u_{t t}-\rho u_{x x}+f\left(\cdot, u_{1}\right)-f\left(\cdot, u_{2}\right) & =0, \\
\rho u_{x}(0, t) & =v(t), \\
u(1, t) & =0, \\
u(x, 0) & =u_{t}(x, 0)=0,
\end{aligned}
$$

in which

$$
\begin{gathered}
v(t)=\sum_{i=1}^{2}(-1)^{i-1} g\left(u_{i}\left(\xi_{0}, t\right)\right)+\int_{0}^{t} k(t-s) \\
\cdot \sum_{i=1}^{2}(-1)^{i-1} h\left(u_{i}\left(\xi_{0}, s\right), \ldots, u_{i}\left(\xi_{N}, s\right)\right) d s .
\end{gathered}
$$

To prove $u_{1}=u_{2}$, then the following lemma is needed.

Lemma 12. Let $u$ be the weak solution of the following problem:

$$
\begin{aligned}
u_{t t}-\rho u_{x x}+F & =0, \quad 0<x<1,0<t<T, \\
u(1, t) & =0 \\
\rho u_{x}(0, t) & =v(t) \\
u(x, 0) & =u_{0}(x), \\
u_{t}(x, 0) & =u_{1}(x), \\
u & \in L^{\infty}(0, T ; H), \\
u_{t} & \in L^{\infty}\left(0, T ; L^{2}(0,1)\right), \\
u(0, \cdot) & \in H^{1}(0, T), \\
v & \in L^{2}(0, T) \\
F & \in L^{1}\left(0, T ; L^{2}(0,1)\right) .
\end{aligned}
$$

Then we have

$$
\begin{aligned}
& \left\|u_{t}(\cdot, t)\right\|^{2}+\rho\|u(\cdot, t)\|_{H}^{2}+2 \int_{0}^{t} v(s) u_{t}(0, s) d s \\
& \quad+2 \int_{0}^{t}\left\langle F(\cdot, s), u_{t}(\cdot, s)\right\rangle d s \geq\left\|u_{1}\right\|^{2}+\rho\left\|u_{0}\right\|_{H}^{2},
\end{aligned}
$$

a.e. $t \in[0, T]$.

Equality holds in case of $u_{0}=u_{1}=0$.

The proof of Lemma 12 is the same as Lemma 2.1 in [16].

Applying Lemma 12 with $u_{0}=u_{1}=0, F=f\left(\cdot, u_{1}\right)-$ $f\left(\cdot, u_{2}\right)$, we get

$$
\begin{gathered}
E(t)=-2 \int_{0}^{t}\left\langle f\left(\cdot, u_{1}\right)-f\left(\cdot, u_{2}\right), u^{\prime}(s)\right\rangle d s \\
-2 \int_{0}^{t} u_{t}(0, s) \sum_{i=1}^{2}(-1)^{i-1} g\left(u_{i}\left(\xi_{0}, s\right)\right) d s \\
-2 \rho u(0, t) \int_{0}^{t} k(t-s) \sum_{i=1}^{2}(-1)^{i-1} \\
\cdot h\left(u_{i}\left(\xi_{0}, s\right), \ldots, u_{i}\left(\xi_{N}, s\right)\right) d s+2 \rho k(0) \\
\cdot \int_{0}^{t} u(0, s) \sum_{i=1}^{2}(-1)^{i-1} \\
\cdot h\left(u_{i}\left(\xi_{0}, s\right), \ldots, u_{i}\left(\xi_{N}, s\right)\right) d s
\end{gathered}
$$




$$
\begin{aligned}
& +2 \rho \int_{0}^{t} u(0, r) d r \int_{0}^{r} k^{\prime}(r-s) \sum_{i=1}^{2}(-1)^{i-1} \\
& \cdot h\left(u_{i}\left(\xi_{0}, s\right), \ldots, u_{i}\left(\xi_{N}, s\right)\right) d s \equiv J_{1}(t)+J_{2}(t) \\
& +J_{3}(t)+J_{4}(t)+J_{5}(t)
\end{aligned}
$$

with $E(t)=\left\|u_{t}(\cdot, t)\right\|^{2}+\rho\|u(\cdot, t)\|_{H}^{2}$.

Now we can estimate the integrals in the right-hand side of (103) as follows.

First term $J_{1}(t)$ : using assumption $\left(A_{3}\right)$, it follows from Lemma 1 and (103) that

$J_{1}(t)$

$$
\begin{aligned}
& =-2 \int_{0}^{t}\left\langle f\left(\cdot, u_{1}(\cdot, s)\right)-f\left(\cdot, u_{2}(\cdot, s)\right), u_{t}(\cdot, s)\right\rangle d s \\
& \leq \frac{d_{M 1}}{\sqrt{\rho}} \int_{0}^{t}\left(\left\|u_{t}(\cdot, s)\right\|^{2}+\rho\|u(\cdot, s)\|^{2}\right) d s \\
& \leq \frac{d_{M 1}}{\sqrt{\rho}} \int_{0}^{t} E(s) d s,
\end{aligned}
$$

where

$$
\begin{aligned}
d_{M 1} & =\left\|D_{2} f\right\|_{C^{0}([0,1] \times[-M, M])}, \\
M & =\sum_{i=1}^{2}\left\|u_{i}\right\|_{L^{\infty}(0, T ; H)} .
\end{aligned}
$$

Second term $J_{2}(t)$ : set $d_{M 2}=\|g\|_{C^{2}([-M, M])}$. Integrating by parts, then we arrive at

$$
\begin{aligned}
& J_{2}(t)=-2 \int_{0}^{t} u_{t}(0, s) \sum_{i=1}^{2}(-1)^{i-1} g\left(u_{i}\left(\xi_{0}, s\right)\right) d s \\
& =-2 \int_{0}^{t} u_{t}(0, s) d s \\
& \quad \cdot \int_{0}^{1} \frac{d}{d \theta} g\left(u_{2}\left(\xi_{0}, s\right)+\theta u\left(\xi_{0}, s\right)\right) d \theta \\
& =-u^{2}(0, t) \int_{0}^{1} g^{\prime}\left(u_{2}\left(\xi_{0}, t\right)+\theta u\left(\xi_{0}, t\right)\right) d \theta \\
& +\int_{0}^{t} u^{2}(0, s) d s \int_{0}^{1} g^{\prime \prime}\left(u_{2}\left(\xi_{0}, s\right)+\theta u\left(\xi_{0}, s\right)\right) \\
& \quad \cdot\left(u_{2}^{\prime}\left(\xi_{0}, s\right)+\theta u^{\prime}\left(\xi_{0}, s\right)\right) d \theta \leq d_{M 2}\left[u^{2}(0, t)\right. \\
& \left.+\int_{0}^{t}\left(\left|u_{1}^{\prime}\left(\xi_{0}, s\right)\right|+\left|u_{2}^{\prime}\left(\xi_{0}, s\right)\right|\right) u^{2}(0, s) d s\right]
\end{aligned}
$$

On the other hand, we easily show that

$$
\|u(\cdot, t)\|^{2} \leq t \int_{0}^{t}\left\|u_{t}(\cdot, s)\right\|^{2} d s \leq T \int_{0}^{t} E(s) d s .
$$

Applying Lemma 2, we deduce from (107) that

$$
\begin{aligned}
u^{2}(0, t) & \leq\|u(\cdot, t)\|_{C^{0}([0,1])}^{2} \\
& \leq \varepsilon\|u(\cdot, t)\|_{H}^{2}+\left(1+\frac{1}{\varepsilon}\right)\|u(\cdot, t)\|^{2} \\
& \leq \frac{\varepsilon}{\rho} E(t)+T\left(1+\frac{1}{\varepsilon}\right) \int_{0}^{t} E(s) d s, \quad \forall \varepsilon>0 .
\end{aligned}
$$

Thus, it follows from (106) and (108) that

$$
J_{2}(t) \leq \varepsilon \frac{d_{M 2}}{\rho} E(t)+\int_{0}^{t} d(s) E(s) d s,
$$

in which

$$
\begin{array}{r}
d(s)=\frac{\varepsilon}{\rho} d_{M 2}\left(\left|u_{1}^{\prime}\left(\xi_{0}, s\right)\right|+\left|u_{2}^{\prime}\left(\xi_{0}, s\right)\right|\right)+\left(1+\frac{1}{\varepsilon}\right) \\
\cdot T d_{M 2}\left(\left\|u_{1}^{\prime}\left(\xi_{0}, \cdot\right)\right\|_{L^{1}(0, T)}+\left\|u_{2}^{\prime}\left(\xi_{0}, \cdot\right)\right\|_{L^{1}(0, T)}+1\right) .
\end{array}
$$

Third term $J_{3}(t)$ : using the Cauchy-Schwartz inequality, we have from (103), $\left(A_{2}\right)$, and $\left(A_{6}\right)$ that

$$
\begin{aligned}
& J_{3}(t)=-2 \rho u(0, t) \int_{0}^{t} k(t-s) \sum_{i=1}^{2}(-1)^{i-1} \\
& \cdot h\left(u_{i}\left(\xi_{0}, s\right), \ldots, u_{i}\left(\xi_{N}, s\right)\right) d s \\
& \quad \leq 2 \sqrt{E(t)} \int_{0}^{t}|k(t-s)| \sum_{i=0}^{N} c_{M i} \sqrt{E(s)} d s \leq \frac{1}{4} E(t) \\
& +4(N+1)^{2} c_{M}^{2} T\|k\|_{L^{\infty}(0, T)}^{2} \int_{0}^{t} E(s) d s .
\end{aligned}
$$

In addition, we can similarly prove as in (111) for the fourth and fifth terms as follows:

$$
\begin{aligned}
& J_{4}(t)=2 \rho k(0) \int_{0}^{t} u(0, s) \sum_{i=1}^{2}(-1)^{i-1} \\
& \cdot h\left(u_{i}\left(\xi_{0}, s\right), \ldots, u_{i}\left(\xi_{N}, s\right)\right) d s \leq 2 \rho|k(0)| \\
& \cdot \int_{0}^{t} \frac{1}{\sqrt{\rho}} \sqrt{E(s)}(N+1) c_{M} \frac{1}{\sqrt{\rho}} \sqrt{E(s)} d s=2(N \\
& +1) c_{M}|k(0)| \int_{0}^{t} E(s) d s, \\
& J_{5}(t)=2 \rho \int_{0}^{t} u(0, r) d r \int_{0}^{r} k^{\prime}(r-s) \sum_{i=1}^{2}(-1)^{i-1} \\
& \cdot h\left(u_{i}\left(\xi_{0}, s\right), \ldots, u_{i}\left(\xi_{N}, s\right)\right) d s \\
& \quad \leq 2 \int_{0}^{t} \sqrt{E(r)} \int_{0}^{r}\left|k^{\prime}(r-s)\right|(N+1) \\
& \cdot c_{M} \sqrt{E(s)} d s d r \leq(N+1) c_{M}\left(\left\|k^{\prime}\right\|_{L^{1}(0, T)}^{2}+1\right) \\
& \cdot \int_{0}^{t} E(s) d s .
\end{aligned}
$$


Choosing $4 \varepsilon d_{M 2} \leq \rho$, the combination of (103), (104), (109), and (111)-(112) shows that

$$
E(t) \leq \int_{0}^{t} \widehat{d}(s) E(s) d s,
$$

where

$$
\begin{aligned}
\widehat{d}(s)= & 2 d(s)+8(N+1)^{2} c_{M}^{2} T\|k\|_{L^{\infty}(0, T)}^{2} \\
& +2(N+1) c_{M}\left(\left\|k^{\prime}\right\|_{L^{1}(0, T)}^{2}+2|k(0)|+1\right) \\
& +2 \frac{d_{M 1}}{\sqrt{\rho}} .
\end{aligned}
$$

By the Gronwall inequality, we see that $E(t) \equiv 0$; that is, $u_{1} \equiv$ $u_{2}$.

This completes the proof of Theorem 5 .

\section{Stability of the Weak Solutions}

In this section, let $\left(u_{0}, u_{1}\right) \in H^{1}(0,1) \times L^{2}(0,1)$ be fixed functions. Also, we assume that $\sigma_{1}, \sigma_{2} \in L^{1}(0,1), \widehat{\sigma} \in$ $L^{2}(0,1), \widehat{f} \in C^{0}(\mathbb{R})$, and $a, b, d, c, c_{M}$ are fixed functions, constants satisfying assumptions $\left(A_{3}\right)-\left(A_{6}\right)$ (independent of $f, g$, and $h$ ). Applying Theorem 5, then problem (1) has a unique weak solution $u$ depending on $\sigma, k, f, g, h$. We denote

$$
u=u(\sigma, k, f, g, h)
$$

where $\sigma, k, f, g, h$ satisfy assumptions $\left(A_{2}\right)-\left(A_{6}\right)$.

Then the stability of the solutions of problem (1) is given as follows.

Theorem 13. Let $\left(A_{1}\right)-\left(A_{6}\right)$ hold. Then the solutions of problem (1) are stable with respect to the data $(\sigma, k, f, g, h)$ in the following sense.

If $\left(\sigma^{j}, k^{j}, f^{j}, g^{j}, h^{j}\right)$ and $(\sigma, k, f, g, h)$ satisfy the assumptions $\left(A_{2}\right)-\left(A_{6}\right)$ such that

$$
\begin{aligned}
\left(\sigma^{j}, k^{j}\right) & \longrightarrow(\sigma, k) \quad \text { strongly in }\left[W^{1,1}(0, T)\right]^{2}, \\
\left(f^{j}, g^{j}, h^{j}\right) & \longrightarrow(f, g, h)
\end{aligned}
$$

strongly in $C^{0}([0,1] \times[-M, M]) \times C^{1}([-M, M]) \times$ $C^{0}\left([-M, M]^{N+1}\right)$, as $j \rightarrow \infty$, for all $M>0$.

Then

$$
\left(u^{j}, u_{t}^{j}\right) \longrightarrow\left(u, u_{t}\right) \quad \text { strongly in } L^{\infty}\left(0, T ; H^{1}(0,1)\right) L^{\infty}\left(0, T ; L^{2}(0,1)\right) \text {, as } j \longrightarrow \infty \text {, }
$$

where $u^{j}=u\left(\sigma^{j}, k^{j}, f^{j}, g^{j}, h^{j}\right), u=u(\sigma, k, f, g, h)$.

Proof of Theorem 13. Firstly, we assume that

$$
\begin{gathered}
\left\|\sigma^{j}\right\|_{W^{1,1}(0, T)}+\|\sigma\|_{W^{1,1}(0, T)} \leq \sigma_{*}, \\
\left\|k^{j}\right\|_{W^{1,1}(0, T)}+\|k\|_{W^{1,1}(0, T)} \leq k_{*},
\end{gathered}
$$

where $\sigma_{*}, k_{*}$ are fixed positive constants.

On the other hand, by the proof of Theorem 5, the a priori estimates of the sequence $\left\{u^{m}\right\}$ satisfy

$$
\begin{aligned}
& \left\|u_{t}^{m}(\cdot, t)\right\|^{2}+\rho\left\|u^{m}(\cdot, t)\right\|_{H}^{2}+\sum_{i=0}^{N} \int_{0}^{t}\left|u_{t}^{m}\left(\xi_{i}, s\right)\right|^{2} d s \\
& \leq C_{T}, \quad \forall t \in[0, T],
\end{aligned}
$$

where $C_{T}$ is a constant depending only on $u_{0}, u_{1}, \sigma_{1}, \sigma_{2}, \widehat{\sigma}$, $f, \widehat{f}, \sigma_{*}, k_{*}, a, b, d, c, \rho, T$.

Due to (119) and (92), we conclude that

$$
\begin{aligned}
C_{T} & \geq \liminf _{m \rightarrow \infty}\left(\left\|u_{t}^{m}(\cdot, t)\right\|^{2}+\rho\left\|u^{m}(\cdot, t)\right\|_{H}^{2}\right. \\
& \left.+\sum_{i=0}^{N} \int_{0}^{t}\left|u_{t}^{m}\left(\xi_{i}, s\right)\right|^{2} d s\right) \geq \liminf _{m \rightarrow \infty}\left\|u_{t}^{m}(\cdot, t)\right\|^{2}+\rho
\end{aligned}
$$

$$
\begin{aligned}
& \cdot \liminf _{m \rightarrow \infty}\left\|u^{m}(\cdot, t)\right\|_{H}^{2}+\sum_{i=0}^{N} \liminf _{m \rightarrow \infty} \int_{0}^{t}\left|u_{t}^{m}\left(\xi_{i}, s\right)\right|^{2} d s \\
& \geq\left\|u_{t}(\cdot, t)\right\|^{2}+\rho\|u(\cdot, t)\|_{H}^{2}+\sum_{i=1}^{N} \int_{0}^{t}\left|u_{t}\left(\xi_{i}, s\right)\right|^{2} d s .
\end{aligned}
$$

In addition, we can prove in a similar way above that the solution $u^{j}$ of problem (1) corresponding to the data $\left(\sigma^{j}, k^{j}, f^{j}, g^{j}, h^{j}\right)$ also satisfies

$$
\begin{aligned}
& \left\|u_{t}^{j}(\cdot, t)\right\|^{2}+\rho\left\|u^{j}(\cdot, t)\right\|_{H}^{2}+\sum_{i=0}^{N} \int_{0}^{t}\left|u_{t}^{j}\left(\xi_{i}, s\right)\right|^{2} d s \\
& \leq \widehat{C}_{T}, \quad \forall t \in[0, T],
\end{aligned}
$$

with $\widehat{C}_{T}$ being a constant depending only on $u_{0}, u_{1}, \sigma_{1}, \sigma_{2}, \widehat{\sigma}$, $f, \widehat{f}, \sigma_{*}, k_{*}, a, b, d, c, \rho, T$.

We set

$$
\begin{aligned}
& \widehat{f_{j}}=f^{j}-f, \\
& \widehat{g_{j}}=g^{j}-g, \\
& \widehat{h_{j}}=h^{j}-h, \\
& \widehat{k_{j}}=k^{j}-k, \\
& \widehat{\sigma_{j}}=\sigma^{j}-\sigma, \\
& v^{j}=u^{j}-u .
\end{aligned}
$$


Then $v^{j}$ satisfies the following problem:

$$
\begin{aligned}
v_{t t}^{j}-\rho v_{x x}^{j}+F^{j} & =0, \quad 0<x<1,0<t<T, \\
v^{j}(1, t) & =0, \\
v_{t}^{j}(0, t) & =w^{j}(t), \\
v^{j}(x, 0) & =v_{t}^{j}(x, 0)=0,
\end{aligned}
$$

where

$$
\begin{aligned}
& F^{j}(x, t)=\widehat{f_{j}}\left(x, u^{j}\right)+f\left(x, u^{j}\right)-f(x, u), \\
& w^{j}(t) \\
& =\widehat{\sigma}_{j}(t)+\widehat{g_{j}}\left(u^{j}\left(\xi_{0}, t\right)\right)+g\left(u^{j}\left(\xi_{0}, t\right)\right) \\
& \quad-g\left(u\left(\xi_{0}, t\right)\right) \\
& \quad+\int_{0}^{t} k^{j}(t-s) \widehat{h_{j}}\left(u^{j}\left(\xi_{0}, s\right), \ldots, u^{j}\left(\xi_{N}, s\right)\right) d s \\
& \quad+\int_{0}^{t} \widehat{k_{j}}(t-s) h\left(u^{j}\left(\xi_{0}, s\right), \ldots, u^{j}\left(\xi_{N}, s\right)\right) d s \\
& \quad+\int_{0}^{t} k(t-s) h\left(u^{j}\left(\xi_{0}, s\right), \ldots, u^{j}\left(\xi_{N}, s\right)\right) d s \\
& \quad-\int_{0}^{t} k(t-s) h\left(u\left(\xi_{0}, s\right), \ldots, u\left(\xi_{N}, s\right)\right) d s .
\end{aligned}
$$

Applying Lemma 12 with $u_{0}=u_{1}=0$, we see that

$$
\begin{aligned}
& E_{j}(t)=-2 \int_{0}^{t}\left\langle f\left(\cdot, u^{j}\right)-f(\cdot, u), v_{t}^{j}(\cdot, s)\right\rangle d s \\
& -2 \int_{0}^{t}\left\langle\widehat{f}_{j}\left(\cdot, u^{j}\right), v_{t}^{j}(\cdot, s)\right\rangle d s-2 \int_{0}^{t} \widehat{\sigma_{j}}(s) \\
& \cdot v_{t}^{j}(0, s) d s-2 \int_{0}^{t} \widehat{g_{j}}\left(u^{j}\left(\xi_{0}, s\right)\right) v_{t}^{j}(0, s) d s \\
& -2 \int_{0}^{t}\left[g\left(u^{j}\left(\xi_{0}, t\right)\right)-g\left(u\left(\xi_{0}, t\right)\right)\right] v_{t}^{j}(0, s) d s \\
& -2 \int_{0}^{t} v_{t}^{j}(0, r) d r \int_{0}^{r} k^{j}(r-s) \\
& \cdot \widehat{h_{j}}\left(u^{j}\left(\xi_{0}, s\right), \ldots, u^{j}\left(\xi_{N}, s\right)\right) d s \\
& -2 \int_{0}^{t} v_{t}^{j}(0, r) d r \int_{0}^{r} \widehat{k_{j}}(r-s) \\
& \cdot h\left(u^{j}\left(\xi_{0}, s\right), \ldots, u^{j}\left(\xi_{N}, s\right)\right) d s
\end{aligned}
$$

$$
\begin{aligned}
& -2\left[\int_{0}^{t} v_{t}^{j}(0, r) d r\right. \\
& \cdot \int_{0}^{r} k(r-s) h\left(u^{j}\left(\xi_{0}, s\right), \ldots, u^{j}\left(\xi_{N}, s\right)\right) d s \\
& -\int_{0}^{t} v_{t}^{j}(0, r) d r \\
& \left.\cdot \int_{0}^{r} k(r-s) h\left(u\left(\xi_{0}, s\right), \ldots, u\left(\xi_{N}, s\right)\right) d s\right] \\
& \equiv K_{1}(t)+K_{2}(t)+\cdots+K_{8}(t),
\end{aligned}
$$

where

$$
E_{j}(t)=\left\|v_{t}^{j}(\cdot, t)\right\|^{2}+\rho\left\|v^{j}(\cdot, t)\right\|_{H}^{2} .
$$

Let $M=\left(\sqrt{C_{T}}+\sqrt{\widehat{C}_{T}}\right) / \sqrt{\rho}$. Now we can estimate eight integrals in the right-hand side of (125) as follows.

Estimating $K_{1}(t)$. From assumption $\left(A_{3}\right)$, it yields

$$
\begin{aligned}
& K_{1}(t) \\
& \quad=-2 \int_{0}^{t}\left\langle f\left(\cdot, u^{j}(\cdot, s)\right)-f(\cdot, u(\cdot, s)), v_{t}^{j}(\cdot, s)\right\rangle d s \\
& \quad \leq \frac{d_{M}}{\sqrt{\rho}} \int_{0}^{t} E_{j}(s) d s,
\end{aligned}
$$

with $d_{M}=\left\|D_{2} f\right\|_{C^{0}([0,1] \times[-M, M])}$.

Estimating $K_{2}(t)$. It is easy to show that

$$
\begin{aligned}
K_{2}(t) & =-2 \int_{0}^{t}\left\langle\widehat{f}_{j}\left(\cdot, u^{j}\right), v_{t}^{j}(\cdot, s)\right\rangle d s \\
& \leq T\left\|\widehat{f}_{j}\right\|_{C^{0}([0,1] \times[-M, M])}^{2}+\int_{0}^{t} E_{j}(s) d s .
\end{aligned}
$$

Estimating $K_{3}(t)$. Since $\rho v_{j}^{2}(0, t) \leq E_{j}(t)$, by CauchySchwartz inequality, then

$$
\begin{aligned}
K_{3}(t)= & -2 \int_{0}^{t} \widehat{\sigma_{j}}(s) v_{t}^{j}(0, s) d s \\
\leq & \varepsilon E_{j}(t)+\frac{1}{\rho}\left\|\widehat{\sigma}_{j}^{\prime}\right\|_{L^{1}(0, T)}+\frac{1}{\varepsilon \rho}\left|\widehat{\sigma_{j}}(t)\right|^{2} \\
& +\int_{0}^{t}\left|\widehat{\sigma}_{j}^{\prime}(s)\right| E_{j}(s) d s .
\end{aligned}
$$

Moreover, using the imbedding $W^{1,1}(0, T) \hookrightarrow C^{0}([0, T])$, there exists a positive constant $k_{T}$ such that

$$
\|v\|_{C^{0}([0, T])} \leq k_{T}\|v\|_{W^{1,1}(0, T)}, \quad \forall v \in W^{1,1}(0, T) .
$$


Thus,

$$
\begin{aligned}
K_{3}(t) \leq & \varepsilon E_{j}(t)+\frac{1}{\rho}\left\|\widehat{\sigma}_{j}\right\|_{W^{1,1}(0, T)}+\frac{k_{T}^{2}}{\varepsilon \rho}\left\|\widehat{\sigma}_{j}\right\|_{W^{1,1}(0, T)}^{2} \\
& +\int_{0}^{t}\left|\widehat{\sigma}_{j}^{\prime}(s)\right| E_{j}(s) d s .
\end{aligned}
$$

Estimating $K_{4}(t)$. By help of assumption $\left(A_{4}\right)$ and (121), we get

$$
\begin{aligned}
K_{4}(t)= & -2 \int_{0}^{t} \widehat{g_{j}}\left(u^{j}\left(\xi_{0}, s\right)\right) v_{t}^{j}(0, s) d s \\
\leq & \varepsilon E(t)+\frac{1}{\rho} \int_{0}^{t} E_{j}(s) d s+\frac{1}{\varepsilon \rho}\left\|\widehat{g}_{j}\right\|_{C^{0}([-M, M])}^{2} \\
& +\left\|\widehat{g}_{j}^{\prime}\right\|_{C^{0}([-M, M])}^{2} \int_{0}^{t}\left|u_{t}^{j}\left(\xi_{0}, s\right)\right|^{2} d s \\
\leq & \varepsilon E(t)+\frac{1}{\rho} \int_{0}^{t} E_{j}(s) d s \\
& +\left(\widehat{C}_{T}+\frac{1}{\varepsilon \rho}\right)\left\|\widehat{g}_{j}\right\|_{C^{1}([-M, M])}^{2}, \quad \forall \varepsilon>0 .
\end{aligned}
$$

Estimating $K_{5}(t)$. Proving in a similar way to (109), we also obtain

$$
\begin{aligned}
& K_{5}(t) \\
& \quad=-2 \int_{0}^{t}\left[g\left(u^{j}\left(\xi_{0}, s\right)\right)-g\left(u\left(\xi_{0}, s\right)\right)\right] v_{t}^{j}(0, s) d s \\
& \quad \leq \frac{\varepsilon}{\rho} E_{j}(t)\|g\|_{C^{2}([-M, M])}+\int_{0}^{t} d_{j}(s) E(s) d s,
\end{aligned}
$$

where

$$
\begin{aligned}
& d_{j}(s)=\|g\|_{C^{2}([-M, M])}\left[\frac{\varepsilon}{\rho}\left(\left|u_{t}^{j}\left(\xi_{0}, s\right)\right|+\left|u_{t}\left(\xi_{0}, s\right)\right|\right)\right. \\
& +\left(1+\frac{1}{\varepsilon}\right) \\
& \left.\cdot T\left(\left\|u_{t}^{j}\left(\xi_{0}, \cdot\right)\right\|_{L^{1}(0, T)}+\left\|u_{t}\left(\xi_{0}, \cdot\right)\right\|_{L^{1}(0, T)}+1\right)\right] .
\end{aligned}
$$

On the other hand, we have

$$
\|v\|_{L^{1}(0, T)} \leq \sqrt{T}\|v\|_{L^{2}(0, T)}, \quad \forall v \in L^{2}(0, T) .
$$

Therefore,

$$
\begin{aligned}
& d_{j}(s) \\
& \leq \frac{\varepsilon}{\rho}\|g\|_{C^{2}([-M, M])}\left(\left|u_{t}^{j}\left(\xi_{0}, s\right)\right|+\left|u_{t}\left(\xi_{0}, s\right)\right|\right) \\
& \quad+T^{3 / 2}\left(1+\frac{1}{\varepsilon}\right)\left(1+\sqrt{C_{T}}+\sqrt{\widehat{C}_{T}}\right)\|g\|_{C^{2}([-M, M])} \\
& =\widehat{d}_{j}(s) .
\end{aligned}
$$

It implies

$$
K_{5}(t) \leq \frac{\varepsilon}{\rho} E_{j}(t)\|g\|_{C^{2}([-M, M])}+\int_{0}^{t} \widehat{d}_{j}(s) E(s) d s .
$$

Estimating $K_{6}(t)$. By reusing the inequalities (121) and (135) and assumption $\left(A_{2}\right)$, then

$$
\begin{aligned}
& K_{6}(t)=-2 \int_{0}^{t} v_{t}^{j}(0, r) d r \\
& \cdot \int_{0}^{r} k^{j}(r-s) \widehat{h_{j}}\left(u^{j}\left(\xi_{0}, s\right), \ldots, u^{j}\left(\xi_{N}, s\right)\right) d s \\
& \leq \varepsilon E_{j}(t)+\frac{2}{\rho} \int_{0}^{t} E_{j}(s) d s+\left(1+T k_{T}^{2}+\frac{1}{\varepsilon \rho}\right) \\
& \cdot\left\|k^{j}\right\|_{W^{1,1}(0, T)}^{2}\left\|\widehat{h_{j}}\right\|_{C^{0}\left([-M, M]^{N+1}\right)}^{2} \leq \varepsilon E_{j}(t)+\frac{2}{\rho} \\
& \cdot \int_{0}^{t} E_{j}(s) d s+k_{*}^{2}\left(1+T k_{T}^{2}+\frac{1}{\varepsilon \rho}\right) \\
& \cdot\left\|\widehat{h}_{j}\right\|_{C^{0}\left([-M, M]^{N+1}\right)}^{2} \cdot
\end{aligned}
$$

Estimating $K_{7}(t)$. Similarly, from assumption $\left(A_{5}\right)$, we also obtain

$$
\begin{aligned}
& K_{7}(t)=-2 \int_{0}^{t} v_{t}^{j}(0, r) d r \\
& \cdot \int_{0}^{r} \widehat{k_{j}}(r-s) h\left(u^{j}\left(\xi_{0}, s\right), \ldots, u^{j}\left(\xi_{N}, s\right)\right) d s \\
& \leq \varepsilon E_{j}(t)+\frac{2}{\rho} \int_{0}^{t} E_{j}(s) d s+\left(1+T k_{T}^{2}+\frac{1}{\varepsilon \rho}\right) \\
& \cdot\left\|\widehat{k}_{j}\right\|_{W^{1,1}(0, T)}^{2}\|h\|_{C^{0}\left([-M, M]^{N+1}\right)}^{2} \equiv \varepsilon E_{j}(t)+\frac{2}{\rho} \\
& \cdot \int_{0}^{t} E_{j}(s) d s+\left(1+T k_{T}^{2}+\frac{1}{\varepsilon \rho}\right) \widehat{d}_{M}\left\|\widehat{k}_{j}\right\|_{W^{1,1}(0, T)}^{2}
\end{aligned}
$$

Estimating $K_{8}(t)$. Due to assumptions $\left(A_{2}\right)$ and $\left(A_{6}\right)$, we get

$$
\begin{aligned}
& K_{8}(t)=-2 \int_{0}^{t} v_{t}^{j}(0, r) d r \\
& \cdot \int_{0}^{r} k(r-s) h\left(u^{j}\left(\xi_{0}, s\right), \ldots, u^{j}\left(\xi_{N}, s\right)\right) d s \\
& +2 \int_{0}^{t} v_{t}^{j}(0, r) d r \\
& \cdot \int_{0}^{r} k(r-s) h\left(u\left(\xi_{0}, s\right), \ldots, u\left(\xi_{N}, s\right)\right) d s \\
& \leq \varepsilon E_{j}(t)+M_{T}^{1} \int_{0}^{t} E_{j}(s) d s,
\end{aligned}
$$


where

$$
\begin{aligned}
M_{T}^{1} & =\frac{1}{\rho}\left[(N+1)^{2} c_{M}^{2}\left(\left\|k^{\prime}\right\|_{L^{1}(0, T)}^{2}+\frac{1}{\varepsilon \rho}\|k\|_{L^{2}(0, T)}^{2}\right)\right. \\
& \left.+2(N+1) c_{M}|k(0)|+1\right] .
\end{aligned}
$$

Thus, combining (125)-(128), (131), (132), and (137)-(140),

$$
\begin{aligned}
E_{j}(t) & \leq \varepsilon M_{T}^{2} E_{j}(t)+M_{T}^{3}\left(\left\|\widehat{f}_{j}\right\|_{C^{0}([0,1] \times[-M, M])}^{2}\right. \\
+ & \left\|{\widehat{g_{j}}}_{j}^{2}\right\|_{C^{1}([-M, M])}^{2}+\left\|\widehat{h}_{j}\right\|_{C^{0}\left([-M, M]^{N+1}\right)}^{2} \\
+ & \left.\left\|\widehat{k}_{j}\right\|_{W^{1,1}(0, T)}^{2}+\left\|\widehat{\sigma_{j}}\right\|_{W^{1,1}(0, T)}^{2}+\left\|\widehat{\sigma}_{j}\right\|_{W^{1,1}(0, T)}\right) \\
& +\int_{0}^{t}\left[\frac{\varepsilon}{\rho}\|g\|_{C^{2}([-M, M])}\left(\left|u_{t}^{j}\left(\xi_{0}, s\right)\right|+\left|u_{t}\left(\xi_{0}, s\right)\right|\right)\right. \\
& \left.+\left|\widehat{\sigma}_{j}^{\prime}(s)\right|+M_{T}^{4}\right] E_{j}(s) d s,
\end{aligned}
$$

in which

$$
\begin{aligned}
M_{T}^{2}= & \frac{1}{\rho}\|g\|_{C^{2}([-M, M])}+5, \\
M_{T}^{3} & \\
= & \left(k_{*}^{2}+\widehat{d}_{M}\right)\left(1+T k_{T}^{2}+\frac{1}{\varepsilon \rho}\right)+\frac{1+k_{T}^{2}+\varepsilon}{\varepsilon \rho}+T \\
& \quad+\widehat{C}_{T}, \\
M_{T}^{4} & \quad \\
= & T^{3 / 2}\left(1+\frac{1}{\varepsilon}\right)\left(1+\sqrt{C_{T}}+\sqrt{\widehat{C}_{T}}\right)\|g\|_{C^{2}([-M, M])} \\
& +\frac{d_{M}}{\sqrt{\rho}}+M_{T}^{1}+\frac{5}{\rho}+1 .
\end{aligned}
$$

Choosing $\varepsilon M_{T}^{2}=1 / 2$ and applying the Gronwall inequality, we deduce from (120), (121), and (142) that

$$
\begin{aligned}
E_{j}( & (t) \leq 2 M_{T}^{3} \exp \left[2 T M_{T}^{4}\right. \\
& \left.+2 \sqrt{T} \frac{\varepsilon}{\rho}\left(\sqrt{C_{T}}+\sqrt{\widehat{C}_{T}}\right)\|g\|_{C^{2}([-M, M])}+2 \sigma_{*}\right] \\
& \cdot\left(\left\|\widehat{f}_{j}\right\|_{C^{0}([0,1] \times[-M, M])}^{2}+\left\|\widehat{g}_{j}\right\|_{C^{1}([-M, M])}^{2}\right. \\
& +\left\|\widehat{h_{j}}\right\|_{C^{0}\left([-M, M]^{N+1}\right)}^{2}+\left\|\widehat{k_{j}}\right\|_{W^{1,1}(0, T)}^{2}+\left\|\widehat{\sigma}_{j}\right\|_{W^{1,1}(0, T)}^{2} \\
& \left.+\left\|\widehat{\sigma}_{j}\right\|_{W^{1,1}(0, T)}\right) .
\end{aligned}
$$

This shows that

$$
\begin{aligned}
& \left\|v_{t}^{j}\right\|_{L^{\infty}\left(0, T ; L^{2}(0,1)\right)}^{2}+\left\|v^{j}\right\|_{L^{\infty}\left(0, T ; H^{1}(0,1)\right)}^{2} \\
& \quad \leq M_{T}\left(\left\|\widehat{f}_{j}\right\|_{C^{0}([0,1] \times[-M, M])}^{2}+\left\|\widehat{g_{j}}\right\|_{C^{1}([-M, M])}^{2}\right. \\
& \quad+\left\|\widehat{h}_{j}\right\|_{C^{0}\left([-M, M]^{N+1}\right)}^{2}+\left\|\widehat{k_{j}}\right\|_{W^{1,1}(0, T)}^{2}+\left\|\widehat{\sigma}_{j}\right\|_{W^{1,1}(0, T)}^{2} \\
& \left.\quad+\left\|\widehat{\sigma}_{j}\right\|_{W^{1,1}(0, T)}\right),
\end{aligned}
$$

with $M_{T}$ being a constant depending only on $u_{0}, u_{1}, \sigma_{1}, \sigma_{2}, \widehat{\sigma}, f, \widehat{f}, \sigma_{*}, k_{*}, a, b, d, c, \rho, T$.

This completes the proof of Theorem 13.

Remark 14. If we use the inequality $\rho v_{j}^{2}\left(\xi_{i}, t\right) \leq E_{j}(t), i=$ $\overline{0, N}$, then, with regard to (126) and (145), conclusion (117) in Theorem 13 can be extended as follows:

$$
\begin{aligned}
& \left(u^{j}, u_{t}^{j}, u^{j}\left(\xi_{0}, \cdot\right), u^{j}\left(\xi_{1}, \cdot\right), \ldots, u^{j}\left(\xi_{N}, \cdot\right)\right) \\
& \quad \longrightarrow\left(u, u_{t}, u\left(\xi_{0}, \cdot\right), u\left(\xi_{1}, \cdot\right), \ldots, u\left(\xi_{N}, \cdot\right)\right),
\end{aligned}
$$

strongly in $L^{\infty}\left(0, T ; H^{1}(0,1)\right) \times L^{\infty}\left(0, T ; L^{2}(0,1)\right) \times$ $\left[C^{0}([0, T])\right]^{N+1}$, as $j \rightarrow \infty$.

\section{Conflict of Interests}

The author declares that there is no conflict of interests regarding the publication of this paper.

\section{References}

[1] M. Aassila, M. M. Cavalcanti, and V. N. Domingos Cavalcanti, "Existence and uniform decay of the wave equation with nonlinear boundary damping and boundary memory source term," Calculus of Variations and Partial Differential Equations, vol. 15, no. 2, pp. 155-180, 2002.

[2] V. Barbu, I. Lasiecka, and M. A. Rammaha, "On nonlinear wave equations with degenerate damping and source terms," Transactions of the American Mathematical Society, vol. 357, no. 7, pp. 2571-2611, 2005.

[3] L. Bociu, P. Radu, and D. Toundykov, "Regular solutions of wave equations with super-critical sources and exponential-tologarithmic damping," Evolution Equations and Control Theory, vol. 2, no. 2, pp. 255-279, 2013.

[4] A. D. Dang and D. P. Alain, "Mixed problem for some semilinear wave equation with a nonhomogeneous condition," Nonlinear Analysis: Theory, Methods \& Applications, vol. 12, no. 6, pp. 581-592, 1988.

[5] T. X. Le and G. Giang Vo, "The shock of a rigid body and a nonlinear viscoelastic bar associated with a nonlinearboundary condition," J. Science Natural Sciences, Uni. Peda. HCM, vol. 8, pp. 70-81, 2006.

[6] L. T. Nguyen and D. T. Bui, "A nonlinear wave equation associated with a nonlinear integral equation involving boundary value," Electronic Journal of Differential Equations, vol. 2004, pp. $1-21,2004$.

[7] L. T. Nguyen, U. V. Le, and T. T. Nguyen, "On a shock problem involving a linear viscoelastic bar," Nonlinear Analysis: Theory, Methods \& Applications, vol. 63, no. 2, pp. 198-224, 2005. 
[8] L. T. Nguyen and G. Giang Vo, "A wave equation associated with mixed nonhomogeneous conditions: global existence and asymptotic expansion of solutions," Nonlinear Analysis: Theory, Methods \& Applications, vol. 66, no. 7, pp. 1526-1546, 2007.

[9] L. T. Nguyen and G. Giang Vo, "A nonlinear wave equation associated with nonlinear boundary conditions: existence and asymptotic expansion of solutions," Nonlinear Analysis: Theory, Methods \& Applications, vol. 66, no. 12, pp. 2852-2880, 2007.

[10] P. Radu, "Weak solutions to the cauchy problem of a semilinear wave equation with damping and source terms," Advances in Differential Equations, vol. 10, no. 11, pp. 1261-1300, 2005.

[11] M. L. Santos, "Asymptotic behavior of solutions to wave equations with a memory condition at the boundary," Electronic Journal of Differential Equations, vol. 2001, no. 73, pp. 1-11, 2001.

[12] D. Takači and A. Takači, "On the approximate solution of a mathematical model of a viscoelastic bar," Nonlinear Analysis: Theory, Methods \& Applications, vol. 67, no. 5, pp. 1560-1569, 2007.

[13] Q. Tiehu, "Global solvability of nonlinear wave equation with a viscoelastic boundary condition," Chinese Annals of Mathematics Series B, vol. 3, 1993.

[14] J. L. Lions, Quelques Méthodes de Résolution des Problèmes aux Limites Non-Linéaires, Dunod, Gauthier-Villars, Paris, France, 1969.

[15] E. Coddington and N. Levinson, Theory of Ordinary Differential Equations, McGraw-Hill, 1955.

[16] J. L. Lions and W. A. Strauss, "Some nonlinear evolution equations," Bulletin de la Société Mathématique de France, vol. 93, p. 79, 1965. 


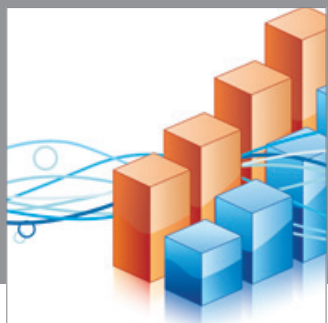

Advances in

Operations Research

mansans

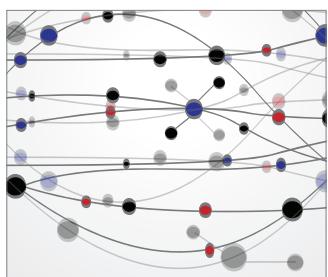

The Scientific World Journal
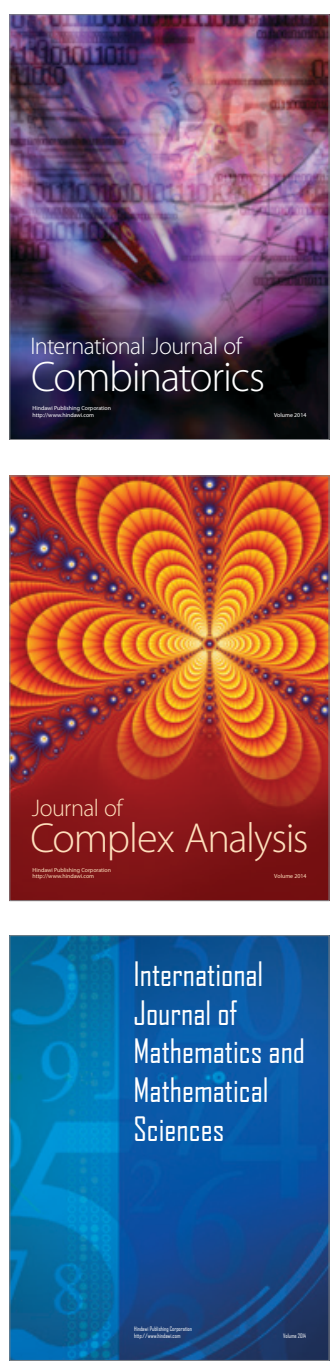
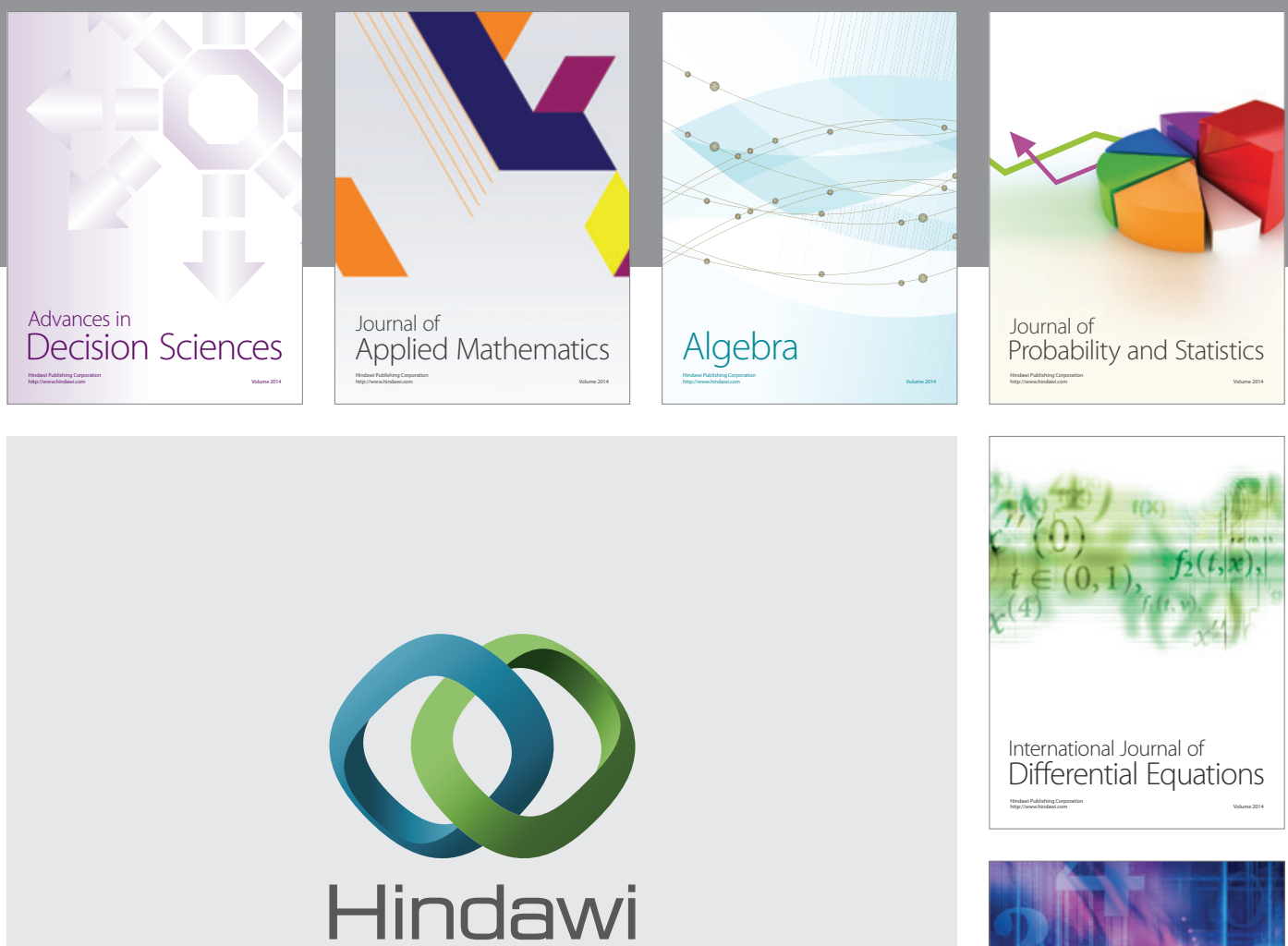

Submit your manuscripts at http://www.hindawi.com
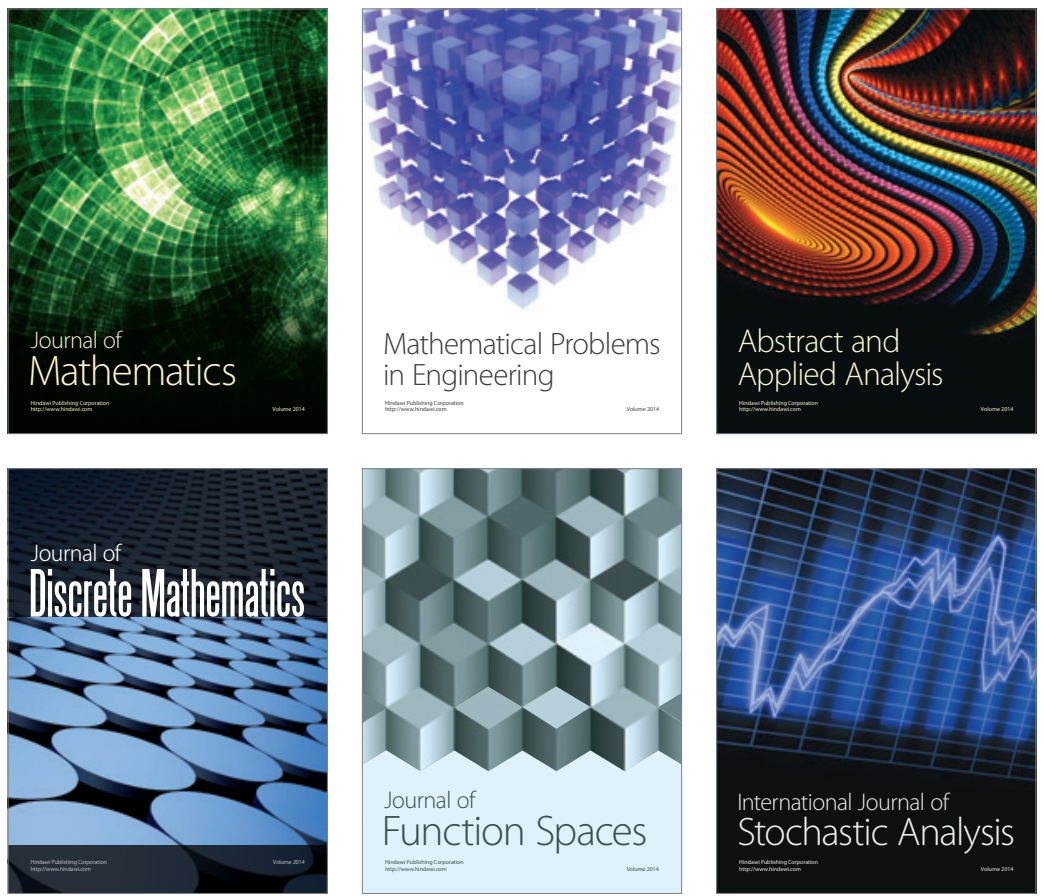

Journal of

Function Spaces

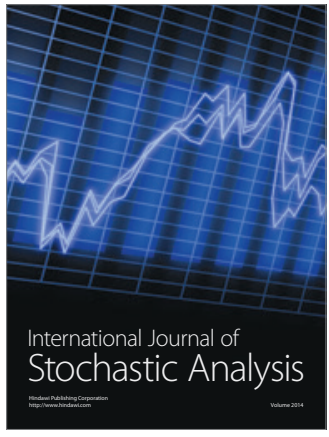

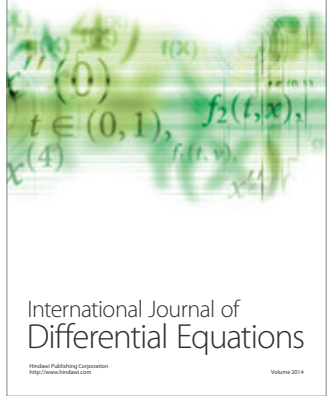
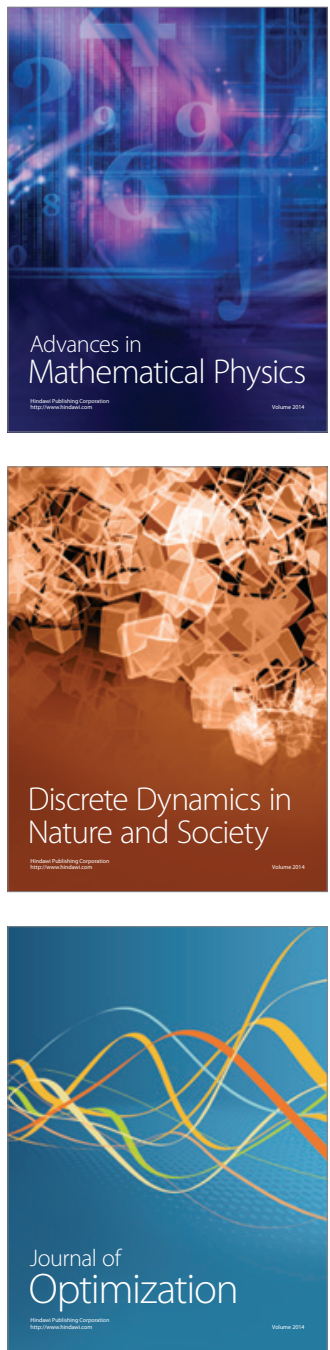